\title{
The SAGE-Spec Spitzer Legacy Program: The Life Cycle of Dust and Gas in the Large Magellanic Cloud
}

\author{
F. Kemper, ${ }^{1}$ Paul M. Woods, ${ }^{1}$ V. Antoniou, ${ }^{2}$ J.-P. Bernard, ${ }^{3}$ R. D. Blum,${ }^{4}$ M. L. Boyer, ${ }^{5}$ J. Chan,,${ }^{6}$ C.-H. R. Chen, ${ }^{7}$ \\ M. Cohen,${ }^{8}$ C. Dijkstra, ${ }^{9}$ C. Engelbracht ${ }^{10}$ M. Galametz,${ }^{11}$ F. Galliano,,${ }^{11}$ C. Gielen, ${ }^{12}$ Karl D. Gordon,${ }^{5}$ \\ V. GorJian, ${ }^{13}$ J. Harris, ${ }^{10}$ S. HonY, ${ }^{11}$ J. L. Hora, ${ }^{14}$ R. Indebetouw,${ }^{7,15}$ O. Jones, ${ }^{1}$ A. KaWAmura, ${ }^{16}$ E. LaGadec,,${ }^{17,1}$ \\ B. Lawton, ${ }^{5}$ J. M. Leisenring, ${ }^{7}$ S. C. Madden, ${ }^{11}$ M. Marengo,${ }^{2,14}$ M. Matsuura,${ }^{18,19}$ I. McDonald, ${ }^{1}$ C. McGuire, ${ }^{1}$ \\ M. Meixner,${ }^{5}$ A. J. Mulia, ${ }^{6}$ B. O'Halloran,${ }^{20}$ J. M. Oliveira,${ }^{21}$ R. Paladini,${ }^{22}$ D. Paradis,${ }^{22}$ W. T. Reach ${ }^{22}$ \\ D. Rubin, ${ }^{11}$ K. Sandstrom,${ }^{8,23}$ B. A. Sargent, ${ }^{5}$ M. Sewilo, ${ }^{5}$ B. Shiao, ${ }^{5}$ G. C. Sloan, ${ }^{24}$ A. K. Speck, ${ }^{6}$ \\ S. Srinivasan, ${ }^{25,26}$ R. Szczerba, ${ }^{27}$ A. G. G. M. Tielens, ${ }^{28}$ E. van Aarle,${ }^{12}$ S. D. VAn DyK, ${ }^{22}$

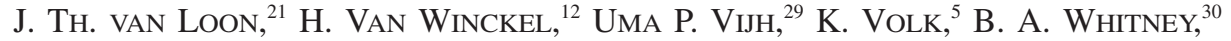 \\ A. N. Wilkins, ${ }^{24}$ AND A. A. ZiJlstra ${ }^{1}$ \\ Received 2010 January 12; accepted 2010 April 6; published 2010 May 19
}

\begin{abstract}
The SAGE-Spec Spitzer Legacy program is a spectroscopic follow-up to the SAGE-LMC photometric survey of the Large Magellanic Cloud carried out with the Spitzer Space Telescope. We present an overview of SAGE-Spec and some of its first results. The SAGE-Spec program aims to study the life cycle of gas and dust in the Large Magellanic Cloud and to provide information essential to the classification of the point sources observed in the earlier SAGE-LMC photometric survey. We acquired $224.6 \mathrm{~h}$ of observations using the infrared spectrograph and the spectral energy distribution (SED) mode of the Multiband Imaging Photometer for Spitzer. The SAGE-Spec data, along with archival Spitzer spectroscopy of objects in the Large Magellanic Cloud, are reduced and delivered to the community. We discuss the observing strategy, the specific data-reduction pipelines applied, and the dissemination of data products to the scientific community. Initial science results include the first detection of an extragalactic $21 \mu \mathrm{m}$ feature toward an evolved star and elucidation of the nature of disks around RV Tauri stars in the Large Magellanic Cloud. Toward some young stars, ice features are observed in absorption. We also serendipitously observed a background quasar, at a redshift of $z \approx 0.14$, which appears to be hostless.
\end{abstract}

Online material: color figures, extended tables

\section{INTRODUCTION}

A photometric survey in the infrared of the Large Magellanic Cloud (LMC) was performed by the Spitzer Legacy Program Surveying the Agents of Galaxy Evolution (SAGE-LMC; Meixner et al. 2006), which charts the budget of gas and dust contributing to the cycle of star formation and stellar death in the Magellanic Clouds. Here we discuss SAGE-Spec, a spectro-

\footnotetext{
${ }^{1}$ Jodrell Bank Centre for Astrophysics, Alan Turing Building, School of Physics and Astronomy, The University of Manchester, Oxford Road, Manchester, M13 9PL, UK.

${ }^{2}$ Department of Physics and Astronomy, Iowa State University, Ames, IA.

${ }^{3}$ Centre d'Étude Spatiale des Rayonnements, 9 Av. du Colonel Roche, BP 44346, 31028 Toulouse cedex 4, France.

${ }^{4}$ NOAO, Tucson, AZ.

${ }^{5}$ Space Telescope Science Institute, Baltimore, MD.

${ }^{6}$ Physics and Astronomy Department, University of Missouri, Columbia, MO.

${ }^{7}$ Department of Astronomy, University of Virginia, Charlottesville, VA.

${ }^{8}$ Radio Astronomy Laboratory, University of California, Berkeley, CA.

${ }^{9}$ Passiebloemweg 31, 1338 TT Almere, The Netherlands.

${ }^{10}$ Steward Observatory, University of Arizona, Tucson, AZ.
}

scopic follow-up to SAGE-LMC, which is also a Spitzer Legacy Program. For SAGE-Spec we observed a variety of circumstellar and interstellar environments with the infrared spectrograph (IRS; Houck et al. 2004) aboard Spitzer (Werner et al. 2004), as well as the SED mode available on the multiband imaging photometer for Spitzer (MIPS; Rieke et al. 2004). The SAGE-Spec data set is exceptionally suited to address the

\footnotetext{
${ }^{11}$ Laboratoire AIM, CEA/DSM-CNRS-Université Paris Diderot DAPNIA/Service d'Astrophysique Bât. 709, CEA-Saclay F-91191 Gif-sur-Yvette Cédex, France.

${ }^{12}$ Instituut Voor Sterrenkunde, KULeuven, Celestijnenlaan 200D, 3001 Leuven (Heverlee), Belgium.

${ }^{13}$ JPL/Caltech, Pasadena, CA.

${ }^{14}$ Harvard-Smithsonian Center for Astrophysics, Cambridge, MA

${ }^{15}$ National Radio Astronomy Observatory, Charlottesville, VA.

${ }^{16}$ Department of Astrophysics, Nagoya University, Chikusa-Ku, Nagoya 464-01, Japan.

${ }^{17}$ ESO Headquarters Garching, Karl-Schwarzschild-Str. 2, D-85748 Garching bei Muenchen, Germany.

${ }^{18}$ Institute of Origins, Department of Physics and Astronomy, University College London, Gower Street, London WC1E 6BT, UK.
} 
following issues: first, it allows us to trace the life cycle of dust and molecular gas on its journey through the galaxy, from dust production sites (asymptotic giant branch [AGB] stars, red supergiants, post-AGB objects, planetary nebulae [PNe]), to the interstellar matter (ISM) (atomic and molecular clouds) to star forming regions (HII regions, young stellar objects); and, second, it allows us to develop a photometric color-color and color-magnitude classification scheme to increase the legacy of the larger SAGE-LMC database. In addition, a large number of smaller astrophysical questions can be addressed using the data set provided here, and a rich harvest in scientific results is expected.

This article gives an overview of the SAGE-Spec Legacy program. We outline the observing strategy, describe the data-reduction process, and discuss the data products that are currently publicly available to the astronomical community (Woods et al. 2010) or will become publicly available in the near future. Existing surveys targeting gas and dust in the LMC are discussed in $\S 2$. This section also includes a description of existing publications of infrared spectroscopy on LMC targets. The observing strategy of the SAGE-Spec project is discussed in $\S 3$, along with a description of the data reduction. This article finishes with some first scientific results of the SAGE-Spec project ( $(4)$, and conclusions and an outlook to the future $(\S 5)$.

\section{SURVEYS: THE GAS AND DUST IN THE LMC}

In order to study the life cycle of dust on a galactic scale, the LMC provides a good compromise between distance and size. It is found at a distance of $\sim 50 \mathrm{kpc}$ (Feast 1999), and as an additional benefit has a favorable viewing angle $\left(35^{\circ}\right.$, van der Marel \& Cioni 2001) resulting in low column densities, and typically just a single interstellar cloud, along each line-of-sight. It is possible to observe individual objects in the LMC due to its vicinity, while at the same time the outside viewpoint that we have enables us to obtain a global view of the LMC through surveys like this.

\footnotetext{
${ }^{19}$ Institute of Origins, Mullard Space Science Laboratory, University College London, Holmbury St. Mary, Dorking, Surrey RH5 6NT, UK.

${ }^{20}$ Astrophysics Group, Imperial College London, Blackett Laboratory, Prince Consort Road, London, SW7 2AZ, UK.

${ }^{21}$ School of Physical and Geographical Sciences, Lennard-Jones Laboratories, Keele University, Staffordshire ST5 5BG, UK.

${ }^{22}$ Spitzer Science Center, California Institute of Technology, MS 220-6, Pasadena, CA.

${ }^{23}$ Max Planck Institut für Astronomie, D-69117 Heidelberg, Germany.

${ }^{24}$ Department of Astronomy, Cornell University, Ithaca, NY.

${ }^{25}$ Department of Physics and Astronomy, Johns Hopkins University, Baltimore, MD.

${ }^{26}$ Institut d'Astrophysique de Paris, 98 bis, Boulevard Arago, Paris 75014, France.

${ }^{27}$ N. Copernicus Astronomical Center, Rabianska 8, 87-100 Torun, Poland.

${ }^{28}$ Leiden Observatory, P.O. Box 9513, NL-2300 RA Leiden, The Netherlands.
}

With $Z \approx 0.3-0.5 Z_{\odot}$, the metallicity of the LMC is subsolar (Westerlund 1997). As a consequence the dust-to-gas mass ratio is $\sim 2-4$ times lower than that in the Solar neighborhood (Gordon et al. 2003), permitting easier penetration of UV radiation to affect physical processes in the ISM and star formation. Indeed, modeling of photon-dominated regions shows that molecular clouds in the LMC will be larger and less dense than clouds in the Milky Way (Pak et al. 1998). The shape of the UV interstellar extinction curve appears to be independent of metallicity, thus constraining the differences between Galactic and LMC grain properties, although large variations exist between environments within the LMC (Misselt et al. 1999).

\subsection{Surveys of the LMC}

Previous infrared surveys of the stellar content of the LMC, performed with, for instance, MSX (Egan et al. 2001) and DENIS (Cioni et al. 2000), are limited to only the brightest sources. Exploration of the stellar content of the LMC was therefore skewed to the tip of the AGB and some bright supergiants. However, with its sensitive arrays, Spitzer has allowed for a full census of all objects brighter than $~ 15$ th magnitude in the $8.0 \mu \mathrm{m}$ band (Meixner et al. 2006). SAGE-LMC encompasses a field of $7^{\circ} \times 7^{\circ}$ covering the majority of the LMC, observed using all bands of the infrared array camera (IRAC; Fazio et al. 2004) and MIPS. The IRAC and MIPS point source catalog contains about 6 million sources and has been made available to the community ${ }^{31}$ (Meixner et al. 2006). The postand pre-main-sequence populations uncovered by SAGE-LMC are discussed by, for example, Blum et al. (2006) and Whitney et al. (2008).

The extended emission component of the SAGE-LMC survey is discussed in more detail by Bernard et al. (2008), who include references to additional LMC surveys in molecular and atomic emission, for example, $\mathrm{H} \alpha$ (Gaustad et al. 2001), $\mathrm{HI}$ (Kim et al. 2003; Staveley-Smith et al. 2003), and CO (Fukui et al. 2008). In addition, a full survey of $\mathrm{OH}$ maser emission in the LMC has been performed (Green et al. 2008), as well as photometric surveys in the optical (e.g., Massey 2002; Zaritsky et al. 2004).

\subsection{Mid-Infrared Spectroscopic Studies}

Several mid-infrared spectroscopic studies of representative targets in object classes in the LMC have already been performed. Most studies focus on individual objects or small samples, although a few systematic studies of large samples exist. Here we provide an overview of studies not including data from SAGE-Spec.

\footnotetext{
${ }^{29}$ Ritter Astrophysical Research Center, University of Toledo, Toledo, OH.

${ }^{30}$ Space Science Institute, Boulder, CO.

${ }^{31}$ The SAGE-LMC point source catalog can be accessed on http://irsa.ipac .caltech.edu/applications/Gator/.
} 


\subsubsection{Silicates}

Prior to the launch of Spitzer, Voors et al. (1999) obtained 2-45 $\mu \mathrm{m}$ spectroscopy of R71, a luminous blue variable in the LMC, using the short wavelength spectrometer (SWS; de Graauw et al. 1996) on board the Infrared Space Observatory (ISO; Kessler et al. 1996). This spectrum contained the first detection of extragalactic crystalline silicates and also showed the presence of polycyclic aromatic hydrocarbons (PAHs).

With Spitzer-IRS the possibility to obtain mid-infrared spectroscopy of individual objects greatly expanded, allowing the analysis of the dust mineralogy. IRS spectroscopy of two $B[e]$ hypergiants (R126 and R66) revealed the presence of silicate dust, where R66 also shows evidence for crystalline silicates and a dual chemistry with the presence of PAHs (Kastner et al. 2006). Both of these sources are known to have disks, which may provide a suitable environment for crystallization. Additional sources found with a silicate mineralogy are IRAS 05003-6712, which shows the characteristic features of crystalline silicates enstatite and forsterite superposed on amorphous silicate features (Zijlstra et al. 2006), and HV 2310, a Mira-type star showing an unusually shaped $10 \mu \mathrm{m}$ resonance, which suggests the presence of both crystalline and amorphous silicates (Sloan et al. 2006a).

\subsubsection{Carbon-Rich and Oxygen-Rich Evolved Stars}

The late-stage AGB stars that dominate the MSX $8 \mu \mathrm{m}$ point source list (Egan et al. 2001) are almost exclusively carbon-rich, as is expected in the low metallicity environment of the LMC (e.g., Zijlstra et al. 2006; Sloan et al. 2008). The $\mathrm{C}_{2} \mathrm{H}_{2}$ molecular absorption bands are deeper than those in their Galactic analogs explained by a higher abundance of this species at low metallicity (Zijlstra et al. 2006). Particularly deep molecular absorption bands are found in IRAS 04496-6958 (Speck et al. 2006), along with a possible detection of $\mathrm{SiC}$ at $11.3 \mu \mathrm{m}$ in absorption. Another object, SMP LMC 11, is classified as a PN based on its emission lines although its infrared SED is more reminiscent of a post-AGB star (Bernard-Salas et al. 2006). The spectrum of SMP LMC 11 shows a variety of organic molecular bands in absorption, including several first extragalactic detections. However, Matsuura et al. (2006) have performed a study of molecular bands $\left(\mathrm{C}_{2} \mathrm{H}_{2}\right.$ and $\left.\mathrm{HCN}\right)$ in a larger sample of carbon stars and found that the abundance of $\mathrm{C}_{2} \mathrm{H}_{2}$ is independent of metallicity, while the $\mathrm{HCN}$ bands remained undetected.

An inventory of dust features in the IRS spectra of carbonrich AGB stars in the LMC is given by Zijlstra et al. (2006) and shows the presence of $\mathrm{SiC}$ and $\mathrm{MgS}$ solid state components. Leisenring et al. (2008) have added a further 19 sources to this sample and devised a method to study the dust condensation sequence in environments of differing metallicity. It was also noted that the occurrence of $\mathrm{MgS}$ correlates with diminished strength of the $\mathrm{SiC}$ feature, suggesting that $\mathrm{MgS}$ forms a coating on $\mathrm{SiC}$ grains (Lagadec et al. 2007; Leisenring et al. 2008), although this seems contradictory with observations of a sample of seven of the most extremely reddened carbon stars (Gruendl et al. 2008), where the presence of $\mathrm{MgS}$ apparently did not hamper the detection of $\mathrm{SiC}$ in absorption.

\subsubsection{Planetary Nebulae}

The prevalence of the carbon-rich phase in stellar evolution is supported by evidence from IRS observations of PNe. Stanghellini et al. (2007) have observed $41 \mathrm{PNe}$ in both Magellanic Clouds, 25 of which are LMC sources, and which were previously observed using the Hubble Space Telescope (HST). Roughly half of the spectra of the LMC PNe are dominated by atomic emission lines, while the remainder show solid state features, of mostly carbon-rich species. Only two LMC PNe show clear detections of oxygen-rich dust, in particular crystalline silicates. An independent study of atomic lines performed on a sample of $25 \mathrm{LMC} / \mathrm{SMC}$ PNe yielded neon and sulfur abundances (Bernard-Salas et al. 2008), both of which are found to be lower than the Galactic values, roughly in the same ratios as the metallicity ratios with respect to the Milky Way.

\subsubsection{Supernovae and Their Remnants}

A detailed study of supernova remnant N132D by Tappe et al. (2006) included IRS observations and showed the emission lines of [NeIII] and [Orv], as well as PAH emission features -including relatively strong emission from the PAH features at $14-20 \mu \mathrm{m}$. The observations were of part of the shell and a fast-moving knot. In supernova remnant $\mathrm{N} 49$ the PAH features are less prominent compared to the atomic emission lines (Williams et al. 2006), which may indicate PAH destruction by UV radiation. The IRS spectrum of recent supernova SN 1987A is dominated by emission from silicate dust, with a few atomic lines (Bouchet et al. 2006). The dust mass derived is $\sim 2.6 \times 10^{-6} M_{\odot}$.

\subsubsection{Pre-Main-Sequence Stars}

IRAS $05328-6827$ is the first YSO to be analyzed with the IRS in the LMC, showing a $\mathrm{CO}_{2}$ ice band that, compared to the $\mathrm{H}_{2} \mathrm{O}$ ice band, is deeper than that typically observed in the Milky Way (van Loon et al. 2005b). Seale et al. (2009) have targeted YSO candidates suggested by Gruendl \& Chu (2009) and spectrally identified 277 YSOs, thus greatly expanding the sample of known YSOs in the LMC. These YSOs were subdivided in six different groups based on the presence of $\mathrm{CO}_{2}$ ice features, silicate features, PAH features, and atomic emission lines.

\subsubsection{Spectral Catalogs of Point Sources}

A number of studies have compiled spectral catalogs. Spanning a range of infrared colors, Buchanan et al. (2006) have 
selected and observed 60 of the brightest $8 \mu \mathrm{m}$ sources and classified 21 red supergiants, 16 carbon-rich and 4 possible oxygenrich AGB stars, $2 \mathrm{OH} / \mathrm{IR}$ stars and the $2 \mathrm{~B}[\mathrm{e}]$ stars discussed in $\S 2.2 .1$. A smaller sample of 28 sources taken from a range of predefined classes is presented by Sloan et al. (2008) and reveals a veritable zoo of spectra. The classification scheme devised by Buchanan et al. (2006) has been used by Kastner et al. (2008) to classify 250 of the most luminous $8 \mu \mathrm{m}$ MSX sources in the LMC, arriving at the conclusion that in this flux-limited sample, carbon-rich AGB stars indeed dominate $35 \%$ of the sources), closely followed by HiI regions (32\%; some of which might contain massive YSOs), and at some distance red supergiants $(18 \%)$. Less prominent in this sample are the populations of oxygen-rich AGB stars (5\%), dusty early-type emission-line stars $(3 \%)$, and foreground AGB stars $(3 \%)$ in this sample. The remaining $4 \%$ of sources could not be classified. This classification was tested by (Buchanan et al. 2009), who found by studying the IRS spectra of objects, which were not previously classified in their earlier work (Buchanan et al. 2006), that 22 out of 31 sources received the correct classification.

\section{OBSERVATIONS}

The Spitzer SAGE-Spec program (PID: 40159) consists of $224.6 \mathrm{hr}$ of spectroscopic observations of targets in the LMC (Table 1). The targets included point sources and extended regions, both of which were observed using the IRS lowresolution and MIPS SED modes. Observations were done in the IRS staring mode for 196 point sources, and 48 point sources were observed in MIPS SED mode. In addition, 10 extended regions were mapped in both the MIPS SED and IRS observing modes. These SAGE-Spec data are discussed in $\S 3.1$ (point sources) and $\S 3.2$ (extended regions).

In addition to the observations made as part of the SAGESpec program, we also deliver to the scientific community our new, homogeneous reductions of all archival IRS and MIPS SED spectroscopic data within the SAGE-LMC footprint, as part of the SAGE-Spec Legacy. Table 2 lists the archival IRS staring mode observations, while the archival IRS maps and

TABLE 1

Summary of Observations in the SAGE-SPEC Program

\begin{tabular}{|c|c|c|}
\hline Observing Mode & Number of Targets & Total Observation Time \\
\hline IRS staring $\ldots \ldots$ & 196 point sources & $108.7 \mathrm{hr}$ \\
\hline \multirow[t]{3}{*}{ IRS mapping } & 10 atomic clouds & $64.2 \mathrm{hr}$ \\
\hline & $10 \mathrm{H}$ II regions & \\
\hline & 20 background & \\
\hline \multirow[t]{3}{*}{ MIPS SED } & 10 atomic clouds & $20.5 \mathrm{hr}$ \\
\hline & $10 \mathrm{H}$ II regions & \\
\hline & 20 background & \\
\hline MIPS SED $\ldots \ldots$ & 48 point sources & $31.2 \mathrm{hr}$ \\
\hline
\end{tabular}

MIPS SED observations within the SAGE-LMC footprint are discussed in $\S \S 3.1 .3$ and 3.2.3.

\subsection{Point Sources}

\subsubsection{Target Selection}

Spectroscopic studies performed with Spitzer prior to the SAGE-LMC survey, in observing cycles 1-3, (e.g., Buchanan et al. 2006; Zijlstra et al. 2006) have predominantly targeted (extreme) AGB stars known before the launch of Spitzer. These objects are concentrated in the brightest part of the [8.0] versus [8.0]-[24] color-magnitude diagram (blue triangles in Fig. 1), above the MSX detection limit.

In order to explore the full life cycle of dust in the LMC and to classify completely the sources in the SAGE-LMC photometric catalogs, we have selected additional sources that cover the range in luminosities and colors found in the SAGE-LMC photometric survey, focusing predominantly on the unexplored region below the MSX detection limit and including some additional bright, extremely red sources (red circles in Fig. 1). The SAGE-Spec program was executed in observing cycle 4 , with the SAGE-LMC data becoming available to the SAGE-LMC/ SAGE-Spec team and the community prior to the proposal deadline for that cycle. Several other proposals targeted point sources in the LMC below the MSX detection limit, most notably the program An Evolutionary Survey of Massive YSOs (PID: 40650; Principal Investigator [PI]: L. Looney; Seale et al. 2009), in which about 300 candidate YSOs were targeted, selected from the SAGE-LMC observations using independent

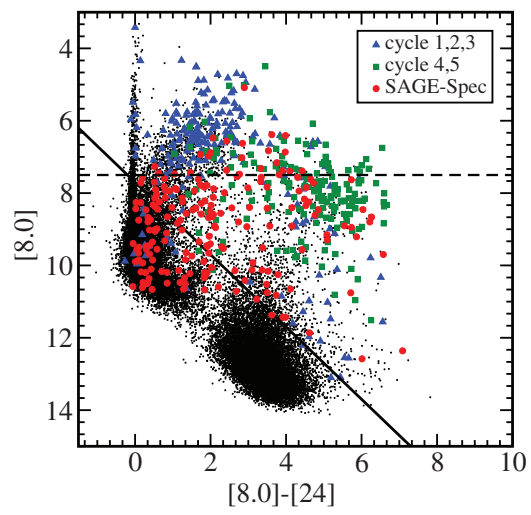

FIG. 1.-[8.0] vs. [8.0]-[24] color-magnitude diagram of infrared point sources in the LMC. The dots represent the sources for which these colors are available in the SAGE-LMC point source catalog (Meixner et al. 2006). Sources selected for observation in the SAGE-Spec program are indicated with circles, whereas archival targets observed in cycles 1-3 and cycles 4-5 are shown as triangles and squares, respectively. The latter group is dominated by targets from PID 40650 (PI: Looney; Seale et al. 2009). The diagonal solid line in the diagram represents the sensitivity limit that we applied to select sources for LL observations. SAGE-Spec sources below this line were only observed with SL. The Midcourse Space Experiment (MSX) detection limit at $8.0 \mu \mathrm{m}$ is shown as a horizontal dashed line. 
TABLE 2

Archival IRS Staring Mode Observations within the SAGE-LMC FootPrint

\begin{tabular}{ccccclc}
\hline \hline AOR Key IRS & R.A. (J2000) Requested & Decl. (J2000) Requested & Proposal ID & PI & AOR Key MIPS SED \\
\hline 10972672 & $\ldots$. & $04 \mathrm{~h} 28 \mathrm{~m} 30.17 \mathrm{~s}$ & $-69 \mathrm{~d} 30 \mathrm{~m} 50.0 \mathrm{~s}$ & 3426 & Kastner & \\
19012352 & $\ldots$. & $04 \mathrm{~h} 32 \mathrm{~m} 57.38 \mathrm{~s}$ & $-69 \mathrm{~d} 26 \mathrm{~m} 33.0 \mathrm{~s}$ & 30788 & Sahai & \\
19012864 & $\ldots$. & $04 \mathrm{~h} 33 \mathrm{~m} 43.69 \mathrm{~s}$ & $-70 \mathrm{~d} 09 \mathrm{~m} 50.0 \mathrm{~s}$ & 30788 & Sahai & \\
19012608 & $\ldots$. & $04 \mathrm{~h} 35 \mathrm{~m} 24.10 \mathrm{~s}$ & $-66 \mathrm{~d} 56 \mathrm{~m} 49.0 \mathrm{~s}$ & 30788 & Sahai & \\
\hline
\end{tabular}

Table 2 is published in its entirety in the electronic edition of the PASP. A portion is shown here for guidance regarding its form and content.

photometry from Gruendl \& Chu (2009); so this area, too, received substantial coverage with IRS over the lifetime of Spitzer. The cycle 4 and 5 targets, dominated by the sample proposed by Seale et al. (2009), are marked with green squares in Figure 1.

For the SAGE-Spec program, we arrived at a target list containing 196 pointings, all of which have been observed using the short low (SL) mode on IRS, while 128 of these were also observed in the long low (LL) mode (see Tables 3 and 4; these tables are published in their entirety in the electronic edition of PASP. A portion is shown here for guidance regarding their form and content). We focused on field stars but also included a small sample of objects from clusters with known metallicities and ages, yielding targets of a much better constrained pedigree than field stars. Cluster stars were mostly overlooked prior to the SAGE-Spec survey. The cluster sources are indicated as such in Table 3.

We selected candidates in a range of object classes. The sample includes candidate AGB stars, both O-rich and C-rich, selected from the work by Srinivasan et al. (2009) and potential YSO sources taken from the list of candidates selected on their colors and magnitudes (Whitney et al. 2008). Because of the overlapping color-magnitude space with YSOs, we also expected to detect background galaxies (Blum et al. 2006). In addition, we ensured representation of rarer objects, such as post-AGB stars and PNe using additional criteria. For the post-AGB stars the samples of Alcock et al. (1998) and Wood \& Cohen (2001) provided a starting point, while the PNe were drawn from lists of LMC PNe assembled by Leisy et al.
(1997) and Reid \& Parker (2006), representing an adequate sampling of electron density and temperature, morphology, and infrared colors. In order to fully cover the colormagnitude space parametrized by IRAC, MIPS, and 2MASS magnitudes, we selected a total of 13 sources from underrepresented regions, such as the region defined by $[3.6]-[8.0]>5$ and the region bordered by $[8.0]>9$ and $J>13.3$. The distribution of the selected sources over the LMC is shown in Figure 2.

\subsubsection{MIPS SED Point Source Target Selection}

We observed 48 point sources with MIPS SED (Tables 2 and 3 ), which is only feasible for the brightest objects in the LMC. A minimum flux level of $100 \mathrm{mJy}$ at $70 \mu \mathrm{m}$ is required to obtain a signal-to-noise ratio $(\mathrm{S} / \mathrm{N})$ of $\sim 3$ for $20 \times 10$ s integrations. We also required all targets selected for MIPS SED observations to have been observed with IRS, either within the context of the SAGE-Spec program or archival programs in cycles 1-3.

\subsubsection{IRS Staring Mode}

\subsubsection{Observations}

The IRS observations of the point sources in the sample are carried out in staring mode. All 196 selected targets were observed using IRS-SL, while the 128 targets with an MIPS$24 \mu \mathrm{m}$ flux $\gtrsim 5.7 \mathrm{mJy}$ were also observed with IRS-LL. Each target was observed in both nod positions, located one-third and two-thirds along the slit. Integration times were chosen

TABLE 3

Point Sources Targeted with the IRS Staring Mode as Part of the SAGE-Spec Program

\begin{tabular}{|c|c|c|c|c|c|c|c|c|}
\hline Number & AOR key IRS & R.A. (J2000) & Decl. (J2000) & SAGE Designation & Modules & Name & AOR Key MIPS SED & Remarks \\
\hline $1 \ldots \ldots$ & 22399232 & $04 \mathrm{~h} 37 \mathrm{~m} 21.15 \mathrm{~s}$ & $-70 \mathrm{~d} 34 \mathrm{~m} 44.57 \mathrm{~s}$ & $\begin{array}{l}\text { SSTISAGEMC } \\
\text { J043721.15-703444.7 }\end{array}$ & SL & $\begin{array}{l}\text { NGC } 1651 \\
\text { SAGE IRS } 1\end{array}$ & & Cluster \\
\hline $2 \ldots \ldots$ & 22399488 & $04 \mathrm{~h} 37 \mathrm{~m} 27.69 \mathrm{~s}$ & $-67 d 54 m 34.94 s$ & $\begin{array}{l}\text { SSTISAGEMC } \\
\text { J043727.61-675435.1 }\end{array}$ & SL, LL & & 22459648 & \\
\hline $3 \ldots \ldots$ & 22399744 & $04 \mathrm{~h} 46 \mathrm{~m} 27.15 \mathrm{~s}$ & $-68 \mathrm{~d} 47 \mathrm{~m} 46.83 \mathrm{~s}$ & $\begin{array}{l}\text { SSTISAGEMC } \\
\text { J044627.10-684747.0 }\end{array}$ & SL, LL & & & \\
\hline $4 \quad \ldots \ldots$ & 22400256 & $04 \mathrm{~h} 47 \mathrm{~m} 18.63 \mathrm{~s}$ & $-69 \mathrm{~d} 42 \mathrm{~m} 20.53 \mathrm{~s}$ & $\begin{array}{l}\text { SSTISAGEMC } \\
\text { J044718.63-694220.6 }\end{array}$ & SL, LL & & & \\
\hline
\end{tabular}

Table 3 is published in its entirety in the electronic edition of the PASP. A portion is shown here for guidance regarding its form and content. 
TABLE 4

Point Sources Targeted with the IRS Staring Mode as Part of the SAGE-Spec Program, Part II

\begin{tabular}{|c|c|c|c|c|c|c|c|c|}
\hline Number & $J$ & $H$ & $K$ & [3.6] & {$[4.5]$} & {$[5.8]$} & {$[8.0]$} & [24] \\
\hline $1 \quad \ldots \ldots \ldots \ldots$ & 12.41 & 11.49 & 11.19 & 10.91 & 10.81 & 10.69 & 10.49 & 9.64 \\
\hline$\ldots \ldots$ & 16.02 & 15.16 & 14.45 & 14.02 & 13.52 & 13.09 & 10.12 & 7.03 \\
\hline $3 \quad \ldots \ldots \ldots \ldots$ & 15.02 & 13.28 & 11.72 & 9.86 & 8.97 & 8.55 & 8.12 & 7.33 \\
\hline $4 \quad \ldots \ldots \ldots \ldots$ & 10.10 & 9.27 & 9.00 & 8.87 & 9.05 & 8.87 & 8.75 & 7.55 \\
\hline
\end{tabular}

Table 4 is published in its entirety in the electronic edition of the PASP. A portion is shown here for guidance regarding its form and content.

${ }^{a}$ MIPS-[24] from nearby LH $\alpha$ 120-N 82, which appears to be the main contributor to the LL spectrum.

based on the IRAC-8.0 and MIPS-24 $\mu \mathrm{m}$ flux levels and targeted to result in an S/N of 60 in SL and 30 in LL, in principle sufficient to analyze and classify dust features on top of a stellar continuum.

Although we observed toward 196 positions, one observation (AOR key 22402560) clearly showed the contribution of two different objects in SL and LL; at short wavelengths the spectrum is due to GV 60, present at the observed location, while the LL spectrum is dominated by nearby Wolf-Rayet star LH $\alpha 120-\mathrm{N} 82$.

\subsubsection{Data-Reduction SAGE-Spec Data}

The processing of data for the SAGE-Spec program began with the flat-fielded images produced by the S18.7 version of the data-reduction pipeline at the Spitzer Science Center (SSC). To extract spectra from the flat-fielded images and cali-

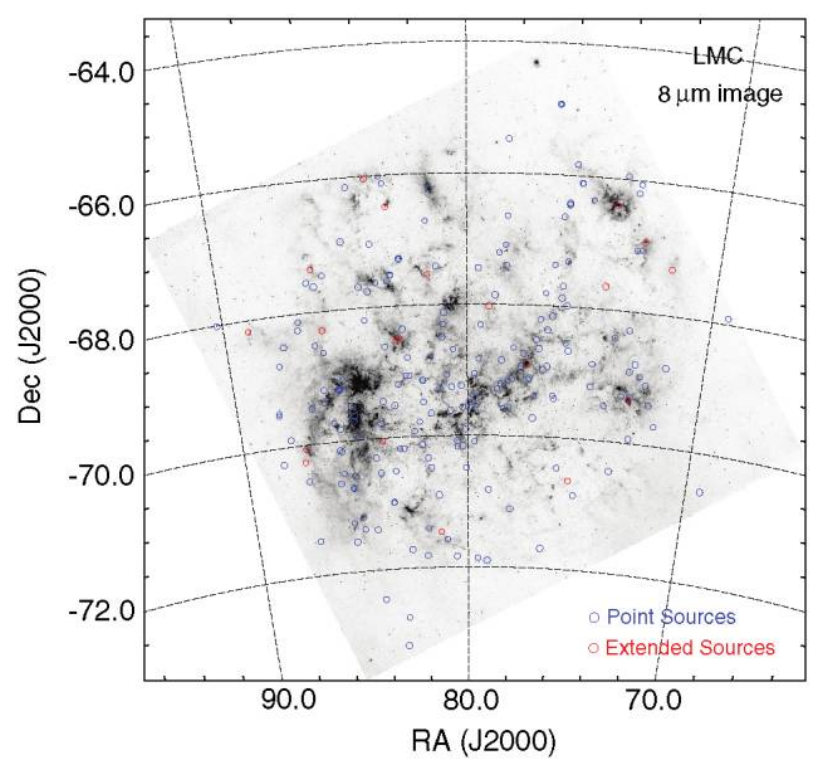

FIG. 2.-Distribution of SAGE-Spec IRS targets over the LMC. The blue symbols indicate the positions of point sources targeted with the IRS staring mode, while the red symbols indicate the central positions of the 20 MIPS SED/IRS maps of extended regions. The positions are overplotted on the IRAC $8 \mu \mathrm{m}$ footprint of the SAGE-LMC survey (Meixner et al. 2006). brate them spectrophotometrically, we followed the procedure used by several other programs in the Magellanic Clouds and elsewhere in the Local Group (e.g,. Sloan et al. 2006b; Zijlstra et al. 2006; Lagadec et al. 2007; Matsuura et al. 2007; Sloan et al. 2008, 2009; Lagadec et al. 2009). The SL and LL modules each have two apertures, one for the second-order data covering the shorter-wavelength portion and the other for the first-order data covering the longer-wavelength portion. When the target is in the second-order aperture (SL2 or LL2), a short piece of first-order data is also obtained, which is referred to as the bonus order (SL-bonus or LL-bonus). The bonus order provides overlap with the true first-order data (SL1 or LL1), making it possible to correct the spectra for discontinuities between the orders.

Observations were constructed so that the number and length of integrations in each aperture within a module matched, giving us flexibility on the background-subtraction method. Generally, in SL, we chose as the background for a given exposure the corresponding exposure with the target in the other aperture. These aperture differences place the positive and negative beams about $79^{\prime \prime}$ apart, compared to $19^{\prime \prime}$ if we had used nod differences. In SL, nod differences would have placed the positive and negative beams close enough to each other to interfere for extended or complex sources. Consequently, we only reverted to nod differences for those observations where the background emission showed a gradient over the 79" throw. For LL, the default for background subtraction was a nod difference, which placed the positive and negative beams $\sim 56^{\prime \prime}$ apart. We generally avoided using aperture differences in LL because the beams were 192" apart, which would often expose us to the more severe background gradients present in the LMC longward of $15 \mu \mathrm{m}$. We examined each image and spectrum carefully to assess when it was necessary to deviate from the default background-subtraction method to avoid either additional sources or complex backgrounds. In some cases, we even reverted to using the image with the target in the other aperture and nod as the background (a cross difference).

In addition to removing the background, differencing the data also corrects most of the rogue pixels in an image. These pixels exhibit dark currents different than their usual levels but generally stable for the duration of a given observation. Some 
pixels remain problematic for a variety of reasons. Most are flagged as such in the rogue pixel masks provided by the SSC. We built super-rogue masks assuming that up to the campaign in which a target was observed, a pixel could be defined as bad if it had been flagged as bad in two previous campaigns. We replaced all flagged pixels using the imclean algorithm developed at Cornell and distributed as a part of irsclean by the $\mathrm{SSC}^{32}$. This algorithm replaces bad pixels by comparing the point-spread functions (PSFs) in adjacent rows.

To extract spectra from the differenced and cleaned images, we used the SSC pipeline modules profile (to locate the source in the slit), ridge (to map the source position and extraction aperture in the image), and extract. ${ }^{33}$ The extract module extracts a spectrum from an image by summing the flux within a pseudorectangle defined for each wavelength element. When the boundaries of a pseudorectangle cross a pixel, the flux is assumed to be evenly distributed within that pixel. The pseudorectangles are centered on the center of the PSF at each wavelength element, and their width increases proportionally with wavelength. The tapered-column extraction within SMART (Higdon et al. 2004) was designed to follow this algorithm precisely, and it gives very similar results.

Spitzer obtains IRS data in a series of data collection events (DCEs). We extracted spectra separately from each DCE, then coadded them to produce one spectrum per nod position. This step produces a mean flux density and a standard deviation, which we divided by the square root of the number of DCEs to estimate the uncertainty in flux density. To calibrate the coadded spectrum from each nod position, we determined spectral corrections using IRS observations of the standard stars HR 6348 (K0 III), HD 166780 (K4 III), and HD 173511 (K5 III). HR 6348 served as the standard for SL (to avoid any difficulties with the strong $\mathrm{SiO}$ absorption in the later $\mathrm{K}$ giants), while all three served as standards for LL (to maximize the $\mathrm{S} / \mathrm{N}$ ). We chose to use K giants rather than $\alpha$ Lac (A1V; e.g., Furlan et al. 2006) because of the difficulty in predicting the strength of the hydrogen recombination lines in the low-resolution modules.

When combining the spectra from the two nod positions for a given order, we replaced the uncertainty when a comparison of the two spectra produced a larger value. When the uncertainty for a given pixel exceeded the average uncertainty in the neighborhood by a factor of 5 (typically), we used only the data from the nod that were closer to neighboring data. This spike-rejection algorithm removed the occasional spikes and divots that survived the previous cleaning step.

Finally, spectra from the 6 orders were combined into $1 \mathrm{spec}-$ trum (SL2, SL-bonus, SL1, LL2, LL-bonus, and LL1; see Table 5). First the bonus-order data were averaged with the first-

\footnotetext{
${ }^{32}$ The irsclean algorithm can be obtained at http://ssc.spitzer.caltech.edu/ dataanalysistools/tools/irsclean/.

${ }^{33}$ These modules are available in SPICE, the Spitzer IRS Custom Extraction package.
}

TABLE 5

WAVELENGTH RANGES

\begin{tabular}{|c|c|}
\hline Segment & Wavelength $(\mu \mathrm{m})$ \\
\hline SL2 & $5.10-7.59$ \\
\hline SL-bonus & $7.23-8.39$ \\
\hline SL1 $\ldots$. & $7.59-14.20$ \\
\hline LL2 & $13.95-20.54$ \\
\hline LL-bonus $\quad \ldots \ldots \ldots \ldots \ldots$ & $19.28-21.23$ \\
\hline LL1 $\ldots \ldots \ldots \ldots \ldots \ldots \ldots$ & $20.46-37.00$ \\
\hline
\end{tabular}

and second-order data where they overlapped and were within the defined range of valid data. Then the spectra were stitched together to remove discontinuities between segments. These discontinuities arose primarily from mispointings, almost always in SL, with its narrower slit (3.6" vs. 10.0"). In general, we assumed that the corrections were always upward to the best-centered spectral segment. All corrections were multiplicative and scalar (i.e., not a function of wavelength). To conclude the processing, we trimmed the spectra of those portions at the ends of each segment that proved impossible to calibrate reliably. We also reset uncertainties that indicated an $\mathrm{S} / \mathrm{N}>500$, as these values are unlikely and can adversely affect algorithms that use the $\mathrm{S} / \mathrm{N}$ to weight the data.

\subsubsection{Archival Data}

We have perused the archive for all staring mode observations within the SAGE-LMC footprint and complemented this list with a few minimap observations, apparently designed to target point sources. The observations are listed in Table 2. Most observations are single pointing staring mode observations but in some cases more complex settings are used. Examples are the minimaps, which appear to be designed to cover the entire PSF, and cluster mode observations, where several pointings are strung together in a single AOR. All archival targets were reduced following the scheme described previously. One target was only observed with SL2, forcing us to depart from our default background subtraction using aperture differences because there is no other aperture to extract. For those data, and in other cases where complex backgrounds made aperture differences inadvisable, we used nod differences in SL.

We include both low resolution (SL and LL) and high resolution ( $\mathrm{SH}$ and $\mathrm{LH}$ ) in the final data delivery. Both short high (SH) and long high ( $\mathrm{LH}$ ) have short slits, which limit the background-subtraction method. Later in the Spitzer mission, the SSC strongly recommended that all $\mathrm{SH}$ and $\mathrm{LH}$ observations include dedicated background observations, but most observations early in the mission came with no background observations. Where these were available, we subtracted them from the images before extracting. When they were not, then we were forced to skip this important step and continue without background subtraction. In all cases, we performed a full-slit extraction, summing all of the flux in the slit at each wavelength. When stitching high-resolution data, we have not applied 
different corrections to the orders within $\mathrm{SH}$ or $\mathrm{LH}$ because these were all obtained simultaneously and cannot differ due to pointing effects. Sample spectra showing the reduced IRS staring mode data are shown in Figure 3.

\subsubsection{MIPS SED Point Sources}

\subsubsection{Observations}

For the MIPS SED mode observations, the integration times are determined based on the measured $70 \mu \mathrm{m}$ emission, from SAGE-LMC, and the slope of the SED. The chop size was chosen to place the background measurement in a region relatively free of emission for the range of dates expected for the observations. Because of the high efficiency of Spitzer scheduling, the MIPS SED observations were taken earlier than expected, resulting in some chop regions not being taken in the ideal locations. The chop sizes were normally $1^{\prime}$, except in five cases were a chop of either $2^{\prime}$ or $3^{\prime}$ was employed. Integration times ranged between 24 and $200 \mathrm{~s}$.

For the LMC, there is one additional point source with MIPS SED observations available in the Spitzer archive. This is SN 1987A and has been observed as part of programs 30067, 40149, and 50444 (PI: Dwek). The IRS spectroscopy and MIPS

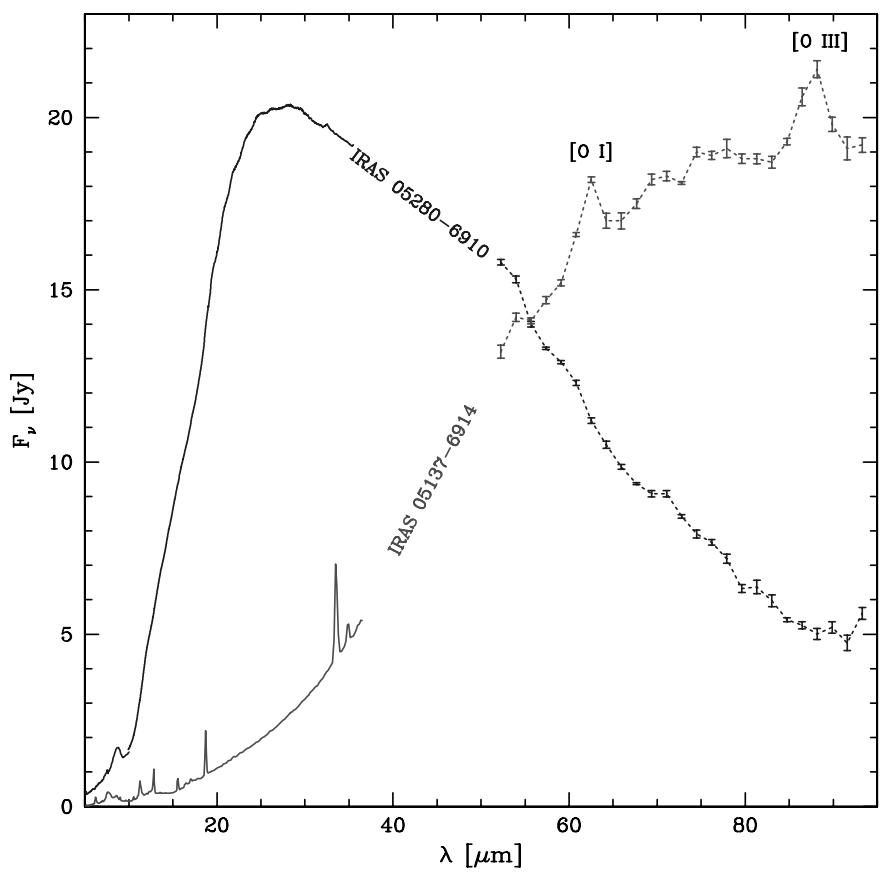

FIG. 3.-Combined MIPS SED (van Loon et al. 2010) and IRS view of two bright, compact IR sources: one, OH/IR star IRAS 05280-6910 is dominated by a warm circumstellar dust envelope with the 10 and $18 \mu \mathrm{m}$ silicate features in absorption and a declining, featureless continuum at FIR wavelengths; the other, ultracompact H II region IRAS $05137-6914$ is dominated by cold dust and an emission-line spectrum both at MIR and FIR wavelengths. See the electronic edition of the PASP for a color version of this figure. and IRAC photometry associated with these programs is already published (Bouchet et al. 2006).

\subsubsection{Data Reduction}

The MIPS SED point source observations were reduced using the MIPS DAT (see Gordon et al. 2005). The extraction was done for a 5 pixel wide aperture centered on the collapsed profile maximum. For the majority of the sources, the off-source chop observations were used to do the initial background subtraction (except when there was significant emission in the offsource chop position). In addition, background subtraction was done by subtracting the measurements made with the same extraction aperture in a region near the edge of the slit. A wavelength dependent aperture correction was applied to extrapolate to infinite extraction aperture. Finally, a smoothed sensitivity function was applied to convert from instrumental to physical units. The uncertainty due to the absolute calibration is $15 \%(\mathrm{Lu}$ et al. 2008). Figure 3 shows the MIPS SED spectrum of point sources IRAS 05280-6910 and IRAS 05137-6914, as examples of the quality of the data. More details on, and first science results from, the MIPS SED observations of point sources in the LMC can be found in van Loon et al. (2010).

\subsection{Extended Regions}

\subsubsection{Target Selection}

For the interstellar extended source observations, we distinguish between highly irradiated (ionized) extended regions (i.e., HII regions) and lower-level irradiated clouds, described as atomic and molecular clouds. The HII regions in the SAGESpec program (see Table 6) have been selected on size and the rms density as derived from the $\mathrm{H} \alpha$ emission (Kennicutt \& Hodge 1986). The source list covers a range in sizes from $1^{\prime}$ to $16^{\prime}$, while the electron density range spans about 2 orders of magnitude. HII regions cover only part of IRAC/MIPS colorcolor space in the LMC (circles in Fig. 4). The atomic regions were selected from the $F_{8.0} / F_{24}$ and $F_{70} / F_{160}$ IRAC/MIPS color-color space, as observed by SAGE-LMC, and are chosen to span a wide range of these IRAC and MIPS colors (Fig. 4). Based on the overall pixel statistics, we divided the color-color space into a number of canonical bins (e.g., $5<F_{8.0} / F_{24}<10$ and $\left.F_{70} / F_{160}<0.1\right)$ and selected regions with high concentrations of pixel values in these bins. As an additional criterion we required these regions to have a distinct identity, for instance, a feature in the Spitzer maps or a CO or H I cloud. The selection is somewhat exploratory in nature due to its small size.

While the HII regions in general tend toward $F_{8} / F_{24}$ ratio $\sim 1$, the atomic regions chosen span higher ratios ranging from 1 to 10 , on average. This selection does not guarantee that HII regions are not present in the atomic regions mapped but samples regions that would be dominated by properties characteristic of atomic ISM. The SAGE-Spec sample has been extended by the archival IRS and MIPS SED maps of the 30 Doradus HII 
TABLE 6

Positions and Map Sizes of Extended Region Observations in the SAGE-Spec Program

\begin{tabular}{|c|c|c|c|c|c|}
\hline Region & AOR Key IRS & AOR Key MIPS SED & R.A. (J2000) & Decl. (J2000) & Size \\
\hline $\mathrm{SSDR}^{\mathrm{a}} 4$ & 22461696 & 22465792 & $04 \mathrm{~h} 47 \mathrm{~m} 40.85 \mathrm{~s}$ & $-67 \mathrm{~d} 12 \mathrm{~m} 31.0 \mathrm{~s}$ & $1.0^{\prime} \times 1.0^{\prime}$ \\
\hline DEM $^{\mathrm{b}}$ L $8 \quad \ldots \ldots$ & 22469120 & 22474240 & $04 \mathrm{~h} 52 \mathrm{~m} 06.31 \mathrm{~s}$ & $-66 \mathrm{~d} 55 \mathrm{~m} 27.1 \mathrm{~s}$ & $1.0^{\prime} \times 1.0^{\prime}$ \\
\hline DEM L $10 \ldots \ldots$ & 22469376 & 22474496 & $04 \mathrm{~h} 52 \mathrm{~m} 11.90 \mathrm{~s}$ & $-69 \mathrm{~d} 20 \mathrm{~m} 43.1 \mathrm{~s}$ & $2.3^{\prime} \times 1.0^{\prime}$ \\
\hline DEM L $34 \quad \ldots \ldots$ & 22469632 & 22474752 & $04 \mathrm{~h} 56 \mathrm{~m} 50.09 \mathrm{~s}$ & $-66 \mathrm{~d} 24 \mathrm{~m} 50.0 \mathrm{~s}$ & $5.4^{\prime} \times 1.0^{\prime}$ \\
\hline DEM L $40 \quad \ldots .$. & 22469888 & 22475008 & $04 \mathrm{~h} 57 \mathrm{~m} 41.21 \mathrm{~s}$ & $-67 \mathrm{~d} 38 \mathrm{~m} 53.9 \mathrm{~s}$ & $2.1^{\prime} \times 1.0^{\prime}$ \\
\hline DEM L $55 \ldots \ldots$ & 22470144 & 22475264 & $05 \mathrm{~h} 01 \mathrm{~m} 41.21 \mathrm{~s}$ & $-70 \mathrm{~d} 38 \mathrm{~m} 48.8 \mathrm{~s}$ & $5.4^{\prime} \times 1.0^{\prime}$ \\
\hline DEM L $86 \quad \ldots \ldots$ & 22470400 & 22475520 & 05h09m55.99s & $-68 \mathrm{~d} 54 \mathrm{~m} 03.2 \mathrm{~s}$ & $4.6^{\prime} \times 1.0^{\prime}$ \\
\hline SSDR $3 \ldots \ldots$ & 22461440 & 22465536 & $05 \mathrm{~h} 15 \mathrm{~m} 43.64 \mathrm{~s}$ & $-68 \mathrm{~d} 03 \mathrm{~m} 20.3 \mathrm{~s}$ & $1.0^{\prime} \times 1.0^{\prime}$ \\
\hline DEM L $188 \ldots \ldots$ & 22470656 & 22475776 & $05 \mathrm{~h} 25 \mathrm{~m} 04.30 \mathrm{~s}$ & $-71 \mathrm{~d} 27 \mathrm{~m} 47.2 \mathrm{~s}$ & $0.5^{\prime} \times 1.0^{\prime}$ \\
\hline SSDR 8 & 22462720 & 22466816 & $05 \mathrm{~h} 26 \mathrm{~m} 25.17 \mathrm{~s}$ & $-67 \mathrm{~d} 29 \mathrm{~m} 08.1 \mathrm{~s}$ & $1.0^{\prime} \times 1.0^{\prime}$ \\
\hline SSDR 11 & 24242176 & $\ldots$ & $05 \mathrm{~h} 31 \mathrm{~m} 07.10 \mathrm{~s}$ & $-68 \mathrm{~d} 19 \mathrm{~m} 12.0 \mathrm{~s}$ & $1.0^{\prime} \times 1.0^{\prime}$ \\
\hline SSDR $1 \ldots \ldots \ldots$ & 22460928 & 22465024 & $05 \mathrm{~h} 32 \mathrm{~m} 02.18 \mathrm{~s}$ & $-68 \mathrm{~d} 28 \mathrm{~m} 13.6 \mathrm{~s}$ & $1.0^{\prime} \times 1.0^{\prime}$ \\
\hline SSDR 9 & 22479360 & 22480384 & $05 \mathrm{~h} 32 \mathrm{~m} 10.73 \mathrm{~s}$ & $-68 \mathrm{~d} 21 \mathrm{~m} 10.8 \mathrm{~s}$ & $1.0^{\prime} \times 1.0^{\prime}$ \\
\hline SSDR 10 & 22479872 & 22480896 & $05 \mathrm{~h} 32 \mathrm{~m} 22.95 \mathrm{~s}$ & $-66 \mathrm{~d} 28 \mathrm{~m} 41.5 \mathrm{~s}$ & $1.0^{\prime} \times 1.0^{\prime}$ \\
\hline SSDR 7 & 22462464 & 22466560 & $05 \mathrm{~h} 35 \mathrm{~m} 09.36 \mathrm{~s}$ & $-70 \mathrm{~d} 03 \mathrm{~m} 24.2 \mathrm{~s}$ & $1.0^{\prime} \times 1.0^{\prime}$ \\
\hline DEM L $243 \ldots$. & 22470912 & 22476032 & $05 \mathrm{~h} 35 \mathrm{~m} 31.10 \mathrm{~s}$ & $-66 \mathrm{~d} 02 \mathrm{~m} 38.8 \mathrm{~s}$ & $5.4^{\prime} \times 1.0^{\prime}$ \\
\hline SSDR $12 \ldots \ldots$ & 24241664 & 24242432 & $05 \mathrm{~h} 43 \mathrm{~m} 39.65 \mathrm{~s}$ & $-68 \mathrm{~d} 46 \mathrm{~m} 18.6 \mathrm{~s}$ & $1.0^{\prime} \times 1.0^{\prime}$ \\
\hline SSDR 2 & 22461184 & 22465280 & $05 \mathrm{~h} 43 \mathrm{~m} 42.01 \mathrm{~s}$ & $-68 \mathrm{~d} 15 \mathrm{~m} 07.4 \mathrm{~s}$ & $1.0^{\prime} \times 1.0^{\prime}$ \\
\hline DEM L $308 \quad \ldots$. & 22471168 & 22476288 & $05 \mathrm{~h} 44 \mathrm{~m} 53.90 \mathrm{~s}$ & $-67 \mathrm{~d} 20 \mathrm{~m} 54.2 \mathrm{~s}$ & $5.4^{\prime} \times 1.0^{\prime}$ \\
\hline SSDR $6 \quad \ldots \ldots \ldots$ & 22462208 & 22466304 & $05 \mathrm{~h} 47 \mathrm{~m} 16.29 \mathrm{~s}$ & $-70 \mathrm{~d} 42 \mathrm{~m} 55.5 \mathrm{~s}$ & $1.0^{\prime} \times 1.0^{\prime}$ \\
\hline DEM L $323 \ldots .$. & 22471424 & 22476544 & $05 \mathrm{~h} 48 \mathrm{~m} 52.39 \mathrm{~s}$ & $-70 \mathrm{~d} 03 \mathrm{~m} 51.5 \mathrm{~s}$ & $3.9^{\prime} \times 1.0^{\prime}$ \\
\hline SSDR $5 \quad \ldots \ldots \ldots$ & 22461952 & 22466048 & $05 \mathrm{~h} 55 \mathrm{~m} 54.19 \mathrm{~s}$ & $-68 \mathrm{~d} 11 \mathrm{~m} 57.1 \mathrm{~s}$ & $1.0^{\prime} \times 1.0^{\prime}$ \\
\hline 30 Dor $^{\mathrm{c}} \quad \ldots \ldots \ldots$ & $\ldots$ & $\ldots$ & $\ldots$ & $\ldots$ & $\sim 7^{\prime} \times \sim 6^{\prime}$ \\
\hline
\end{tabular}

region (Indebetouw et al. 2009) and two additional atomic regions in the LMC from PID 40031 (PI: G. Fazio; SSDR 11 and SSDR 12 in Table 6), of which the data remain unpublished so

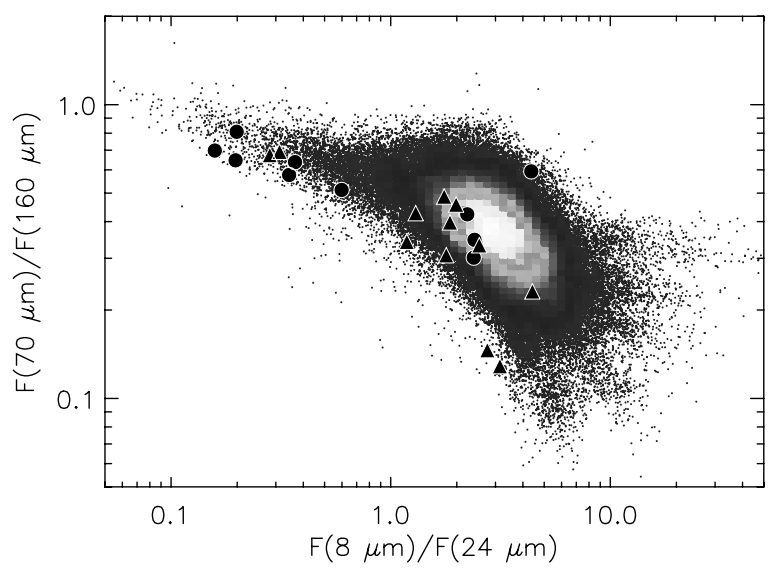

FIG. 4.-IRAC/MIPS color-color diagram for extended regions. The SAGELMC data for all three MIPS bands and the IRAC $8 \mu \mathrm{m}$ band were convolved to the spatial resolution of the MIPS- $160 \mu \mathrm{m}$ band. The background dots represent the ratios of these bands for each MIPS-160 spatial resolution element. The H II regions that were observed for the SAGE-Spec program are plotted as circles and the diffuse regions as triangles. See the electronic edition of the PASP for a color version of this figure. far. The central positions and sizes of all 23 extended regions are listed in Table 6.

\subsubsection{IRS Mapping Mode}

\subsubsection{Observations}

For the atomic and molecular cloud observations, our sensitivity objective was to obtain spectral maps such that when spatially integrated over a $1^{\prime} \times 1^{\prime}$ region, we would achieve an $\mathrm{S} / \mathrm{N}=10$. We used exposure times per pixel of $4 \times 14 \mathrm{~s}$ $(\mathrm{SL})$ and $4 \times 30 \mathrm{~s}(\mathrm{LL})$ and spectral mapping to cover each $1^{\prime} \times$ $1^{\prime}$ region. All selected HII regions are mapped in strips that have a width of $1^{\prime}$, and the length being the diameter of the HII region. The mapping is done in such a way that the SL slit is stepped in the cross-slit direction by the diameter of the HII region and contains two pointings in the slit direction. The LL slit is stepped in the cross-slit direction by $1^{\prime}$ and again in the slit direction by the diameter of the HII region, thus obtaining a $1^{\prime}$ wide strip in both LL and SL. There are four $6 \mathrm{~s}$ exposures for both modules, and, because the maximum length of an IRS AOR is $6 \mathrm{~h}$, the total length of the strip is limited to 5.4'. The largest HII regions in our sample are therefore not mapped to their full diameter. For both the low surface brightness clouds and the HII regions, dedicated off-source observations were obtained to remove the time dependent IRS detector hot pixels and zodiacal light background. 


\subsubsection{Data Reduction}

We used the standard pipeline data as produced by the SSC. The individual observations were combined into a spectral cube using CUBISM (Smith et al. 2007a), and these spectral cubes were merged together using custom software (Sandstrom et al. 2009). Each independent spectrum in the cube was fit using PAHFIT (Smith et al. 2007b) after convolution to a common resolution using custom convolution kernels (Gordon et al. 2008; Sandstrom et al. 2009). The fit parameters are used to construct the feature maps. For the molecular and atomic regions, we did achieve an $\mathrm{S} / \mathrm{N}$ of 10 , especially at $\lambda>10 \mu \mathrm{m}$. For the HII regions, more mixed results emerged, with only 6 of the 10 regions meeting the $\mathrm{S} / \mathrm{N}$ goal. In those regions with an $\mathrm{S} / \mathrm{N}>10$ it is possible for us to investigate spatial variations in the properties of dust and PAHs and correlate this with the interstellar radiation field measured through PAH feature strengths and atomic line ratios.

We have also spatially integrated the IRS (and MIPS SED) spectroscopy, as Figure 5 shows for one of the atomic regions. The integrated SED of this region was extracted from the individual IRS order cubes and MIPS SED cube over the region in common between all the observations. In this example, the extracted region was a $60^{\prime \prime}$ diameter circle. When studying spatial variations is not possible due to low $\mathrm{S} / \mathrm{N}$, the integrated spectra still yield global information on the dust and PAH properties, and the radiation fields in these environments.

\subsubsection{MIPS SED}

\subsubsection{Observations}

For the HII regions, the MIPS SED observations roughly coincide with the peak of the SED and pick up any strong [OI] $63 \mu \mathrm{m}$ and [OIII] $88 \mu \mathrm{m}$ lines (Indebetouw et al. 2009). For the atomic and molecular regions the MIPS SED observations constrain dust temperatures, and, in particular, the very small grain emission properties. All SAGE-Spec extended regions are mapped with one-half slit offsets in both slit dimensions (92009 cross slit and 1.25" along slit), with the minimum exposure time of $3 \mathrm{~s}$. With these exposure times, we aimed to achieve an $\mathrm{S} / \mathrm{N}$ of 5 per spatial bin for the HII regions, while for the diffuse regions, the objective is for an $\mathrm{S} / \mathrm{N}$ of 5 for the spatially integrated SED $\left(1^{\prime} \times 1^{\prime}\right.$ region $)$. Indeed, these S/ $\mathrm{N}$ goals were met for 7 out of $10 \mathrm{HII}$ regions and all of the diffuse regions. For all extended regions, dedicated background observations off the LMC were obtained.

For the LMC, there are two additional extended sources with MIPS SED observations available in the Spitzer archive. They are 30 Dor (Indebetouw et al. 2009) and the $70 \mu \mathrm{m}$ excess (as described by Bernard et al. 2008) region observed in PID 40031, for both of which IRS mapping mode observations are also available (see $\S 3.2 .2$ ).

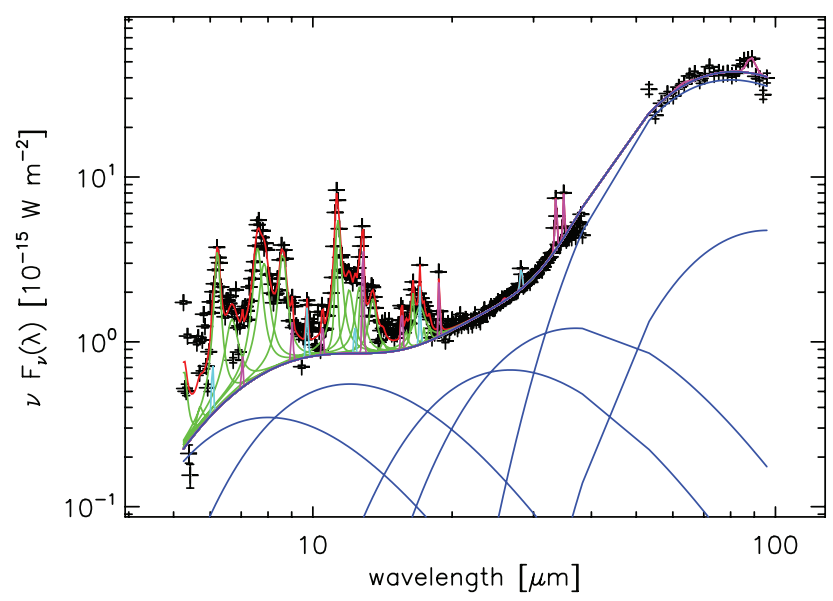

FIG. 5.-Integrated IRS and MIPS SED spectrum of SSDR 1. The spectrum is spatially integrated over a circle with diameter $60^{\prime \prime}$, centered on the position listed in Table 6. A modified form of the PAHFIT model has been applied (see main text). The plus signs indicate the data, and the solid lines are the various components of the PAH fit model, with blue representing the dust continuum, cyan, the atomic transitions, and green, the PAH bands. The overall fit is indicated with a red line.

\subsubsection{Data Reduction}

The MIPS SED extended source observations were reduced using the MIPS DAT v3.10 (Gordon et al. 2005), in a way similar to MIPS imaging data (see, e.g., Dale et al. 2007), and calibrated according to the prescription of Lu et al. (2008). Using the MIPS DAT we constructed three different products: onsource background subtracted, on-source only, and off-source only rectified mosaics combining all the appropriate observations in an AOR. The dedicated off-LMC background observations were subtracted from the on- and off-source mosaics

For each spectral map, and using the on-source and offsource MIPS DAT products, spectral cubes were populated by assuming that the slit is 2 pixels wide and has the coordinates and orientation found in the header of each input image. For any pixel in the output cube where multiple input values are available, the output value is the mean of the input values, weighted by the inverse square of the uncertainty associated with that value. This procedure has been captured in custom IDL software, which parallels that of the similar software for IRS spectra (CUBISM; Smith et al. 2007a). An example of the resulting spectra, integrated over a larger region, is shown in Figure 5.

\subsection{Data Products and Dissemination}

As part of the SAGE-Spec Legacy program we deliver basic and enhanced data products to the astronomical community. ${ }^{34}$ Reduced spectra for all point sources and extended sources

\footnotetext{
${ }^{34}$ The SAGE-Spec data products are available on http://data.spitzer.caltech .edu/popular/sage-spec/. The unprocessed data are also available through the SSC archive tool Leopard
} 
within the SAGE-LMC footprint are included in the delivery in the form of tables and plots. This data set includes both the SAGE-Spec and archival observations within this footprint. The enhanced data products include a spectral catalog of the archival and the SAGE-Spec point sources, along with a source classification. In addition, a photometric classification scheme will be derived and applied to the entire SAGE-LMC point source catalog.

In the cases where spectral maps were performed on extended sources, the data will be spatially averaged into one spec- trum. We also deliver spectral data cubes for spectral maps. From those data cubes, maps in selected features or spectral lines will be provided for extended regions with sufficient flux in the intended features. Figure 6 shows an example of these spectral feature maps.

The first delivery took place on 2009 August 1 and included the reduced data of all SAGE-Spec IRS staring mode observations, along with a first batch of MIPS SED point source observations (Woods et al. 2010). The second delivery in March 2010 contains the reduced spectral data cubes for the extended
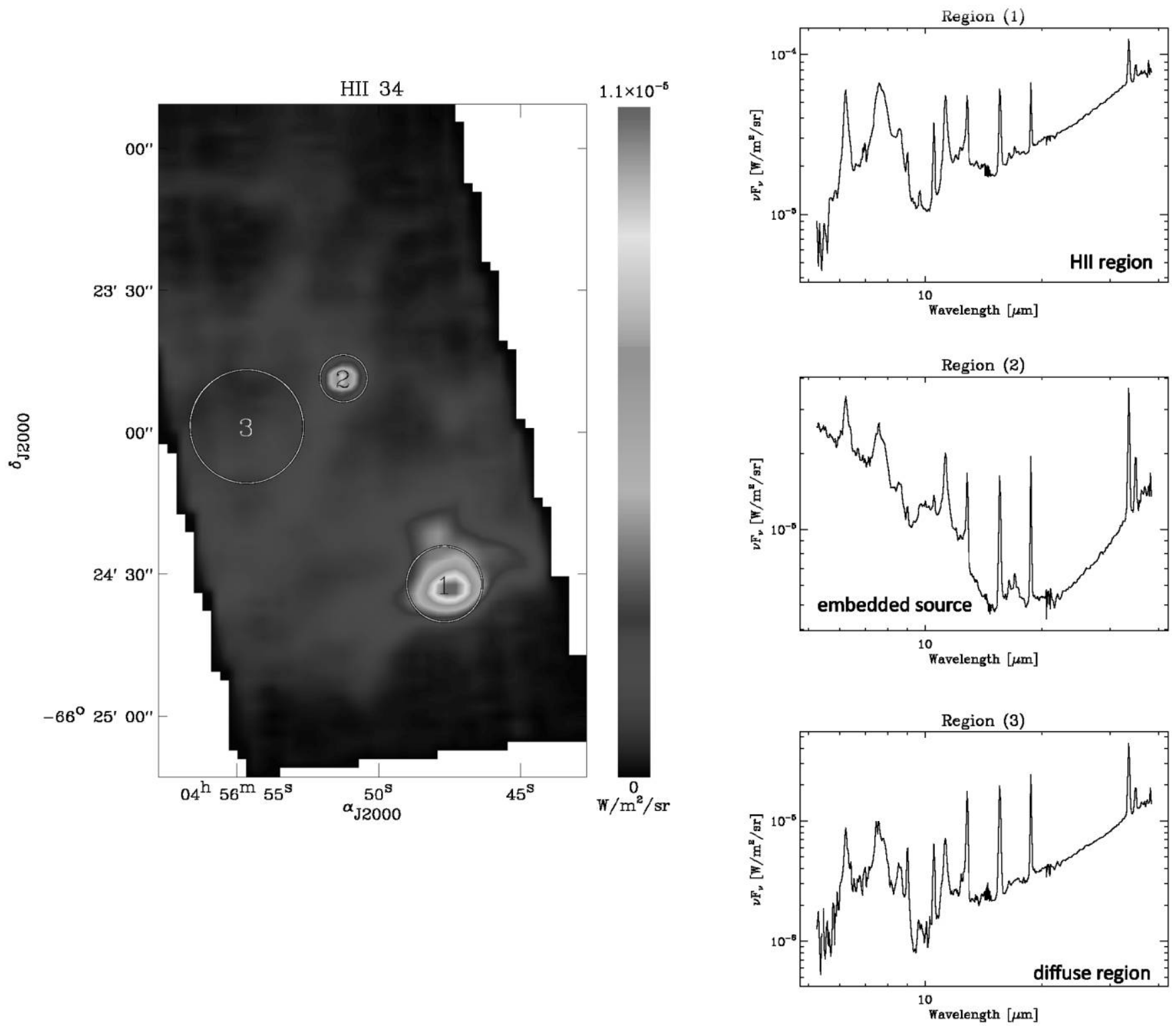

FIG. 6.-Example of a spectral map of an $\mathrm{H}$ II region (LHA 120-N 11; DEM L 34) and its surroundings. The map shows the integrated intensity between 6-9 $\mu$ m for the region where there is full coverage over the entire IRS LL and SL range. The panels on the right show the extracted spectra from different regions in the map. Region 1 is centered on N11B, a compact HII region. Its IRS spectrum shows nebular line and PAH emission on a steeply rising IR continuum. Region 2 is centered on an embedded cluster that shows a strong stellar continuum together with an IR excess due to the surrounding dust. Region 3 represents a more diffuse region, and it exhibits very prominent nebular lines in its IRS spectrum. (S. Hony et al. 2010, in preparation). See the electronic edition of the PASP for a color version of this figure. 
regions observed with MIPS SED and IRS (both SAGE-Spec and archival data) and the reduced archival IRS staring mode spectroscopy from Cycles 1-3 (Woods et al. 2010). Two more deliveries are planned at $\sim 6$ month intervals, encompassing archival data from cycles 4 and 5 and the enhanced data products.

\section{FIRST RESULTS}

Here, we list some first scientific results of the SAGE-Spec collaboration, in the context of both program aims: following the life cycle of gas and dust $(\S 4.1)$ and the classification of point sources $(\S 4.2)$.

\subsection{Life Cycle of Gas and Dust}

\subsubsection{Evolved Stars}

\subsubsection{Carbon-Rich Post-AGB Stars}

Four carbon-rich post-AGB objects in the combined SAGESpec and archival samples can easily be identified by their spectral characteristics: they have strong PAH emission and exhibit the $30 \mu \mathrm{m}$ feature generally attributed to $\mathrm{MgS}$ grains. In addition, the dust temperature is clearly low compared to that in carbon stars still on the AGB as judged from the shape of the continuum. These objects most likely have left the AGB within the last few hundred years and are evolving to become PNe. The IRS spectra of the four objects along with the available pho- tometry in the literature are presented in Figure 7. Three of the objects are SAGE-Spec targets and selected as likely post-AGB candidates, while object NGC 1978 WBT 2665 was part of the sample of PID 3591 (Table 2), and selected on its infrared colors using the classification by Egan et al. (2001).

IRAS F05192-7009 shows a strong $21 \mu \mathrm{m}$ feature, so far only observed in post-AGB objects in our Galaxy (see, e.g., Hrivnak et al. 2009 and references therein), thus representing the first extragalactic detection of this feature. In the other three objects it is weak or absent. For object SAGE1C J051228.18 -690755.7 the data around the position of the feature possibly contain an artifact, so it is possible that there is a $21 \mu \mathrm{m}$ feature present, but if so it is quite weak and it seems more likely that the feature is absent. Preliminary dust radiative transfer modeling for IRAS 05192-7009 shows that the $21 \mu \mathrm{m}$ and $30 \mu \mathrm{m}$ feature shapes are the same as those observed in Galactic objects and suggests that the bulk of the dust in the circumstellar shell is hotter than that found for the Galactic $21 \mu \mathrm{m}$ sources. The models indicate that the dust shell for IRAS 05192-7009 is somewhat more massive than those of a number of the well studied Galactic $21 \mu \mathrm{m}$ objects such as IRAS 07134+1005 and IRAS $22272+5435$. The same may be the case for all four objects, which would then suggest that they have left the AGB more recently than most of or all of the Galactic $21 \mu \mathrm{m}$ objects.

Compared to Galactic objects of this type, the LMC sources show very strong PAH emission features, including the rarely seen $6.9 \mu \mathrm{m}$ feature. Comparison of the IRAC colors of these
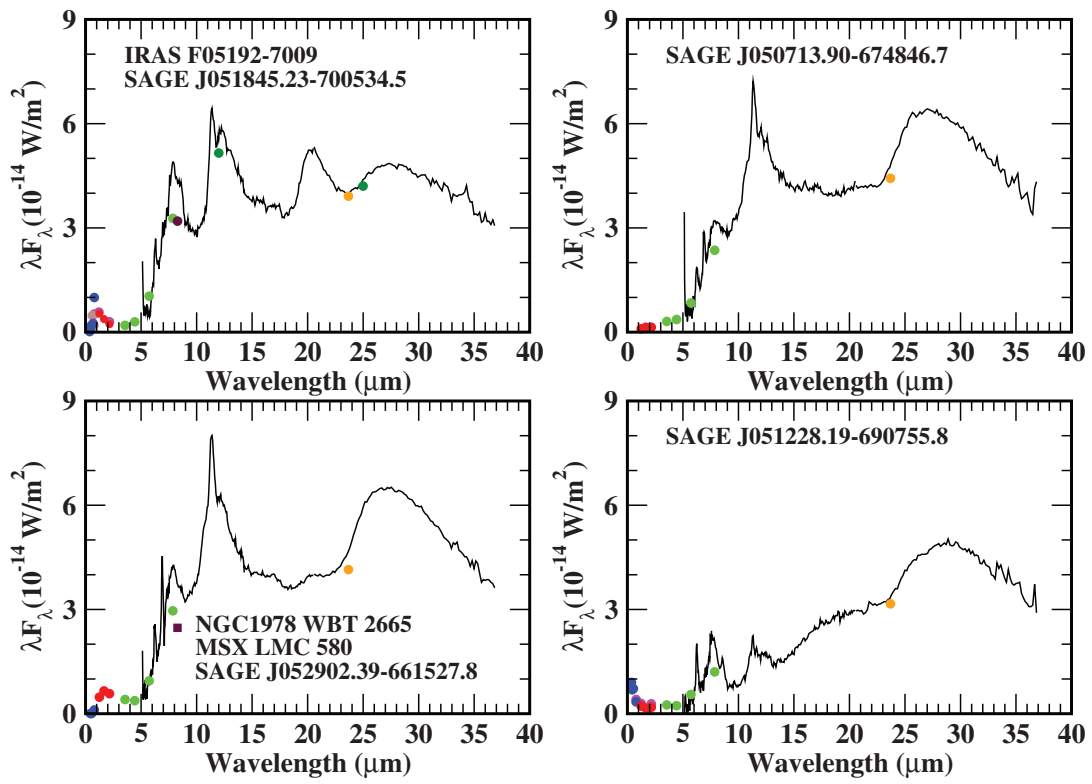

FIG. 7.-IRS spectra of the four post-AGB candidate objects are given all on the same scale for direct comparison. In each panel the available photometry from the literature is also plotted: green for the SAGE-LMC IRAC photometry, orange for SAGE-LMC MIPS-24 photometry, red for 2MASS photometry (Skrutskie et al. 2006; Cohen et al. 2003), maroon for MSX (from the reject catalog for NGC 1978 WBT 2665; Price et al. 2004), blue for the Magellanic Cloud Photometric Survey photometry (Zaritsky et al. 1997), magenta for DENIS photometry (Fouqué et al. 2000), dark green for IRAS photometry (Beichman et al. 1988), and brown/cyan for Palomar Sky Survey photometry (Monet et al. 2003). No scaling has been applied to any of the data values. 
objects to the simulated IRAC/MIPS colors of Galactic $21 \mu \mathrm{m}$ objects with good quality ISO SWS data indicates that the [3.6][8.0] colors for the LMC objects are larger than those for the Galactic objects, which may be due to some combination of being less evolved off the AGB and having the strong PAH features. All of these objects have a well-defined position in a K-[8.0] versus K-[24] color-color diagram, which suggests that further candidate post-AGB objects can be identified from the SAGE-LMC data.

\subsubsection{RV Tauri Stars}

A specific subclass of post-AGB stars is formed by the RV Tauri stars. These are pulsating stars that occupy the highluminosity tail of the population II Cepheids. Their light curves are characterized by a succession of deep and shallow minima. Many objects also show significant cycle-to-cycle variability.

One of the more remarkable properties of RV Tauri stars is that the observed chemical pattern in the photospheres of many Galactic RV Tauri stars is the result of a chemical rather than a nucleosynthetic process: the photospheres are found to be deficient in refractory elements (like $\mathrm{Fe}$ and $\mathrm{Ca}$ and the s-process elements), while the nonrefractory elements are not (or much less) affected (Giridhar et al. 2005; Maas et al. 2005 and references therein). The photospheric patterns can be understood by a process in which gas-dust separation is followed by reaccretion of only the gas, which is poor in refractory elements. This process has likely only taken place in systems that are surrounded by stable dusty disks (Waters et al. 1992), which RV Tauri stars are known to possess (van Winckel 2003).

These dusty disks around evolved objects are ideal environments to foster strong grain processing, and in a Spitzer survey of 21 Galactic sources Gielen et al. (2008) showed that very high crystallinity prevails and is dominated by magnesium-rich end members of olivine and pyroxene silicates. RV Tauri stars in the LMC were found by the microlensing survey MACHO (Alcock et al. 1998), and high-resolution optical spectroscopy revealed that depletion of refractory elements is also observed in their photospheres (Reyniers \& van Winckel 2007).

Using SAGE-Spec data, we showed that also in the LMC, the RV Tauri stars have stable disks, rather than dusty outflows (Gielen et al. 2009), and the close connection between photospheric depletion and the stable dusty environment is illustrated in Figure 8.

\subsubsection{Interstellar Medium}

The integrated spectrum of SAGE-Spec diffuse region 1, hereafter SSDR 1, which is also known as CO cloud 154 from the NANTEN CO survey (Fukui et al. 2008), or LMC N J05316830 , with a mass (estimated from the CO) of $1.0 \times 10^{6} M_{\odot}$ (Fukui et al. 2008), is shown in Figure 5. The spectrum is dominated by PAH features at $5-15 \mu \mathrm{m}$ and a continuum from small grains at longer wavelengths, while big grains dominate the

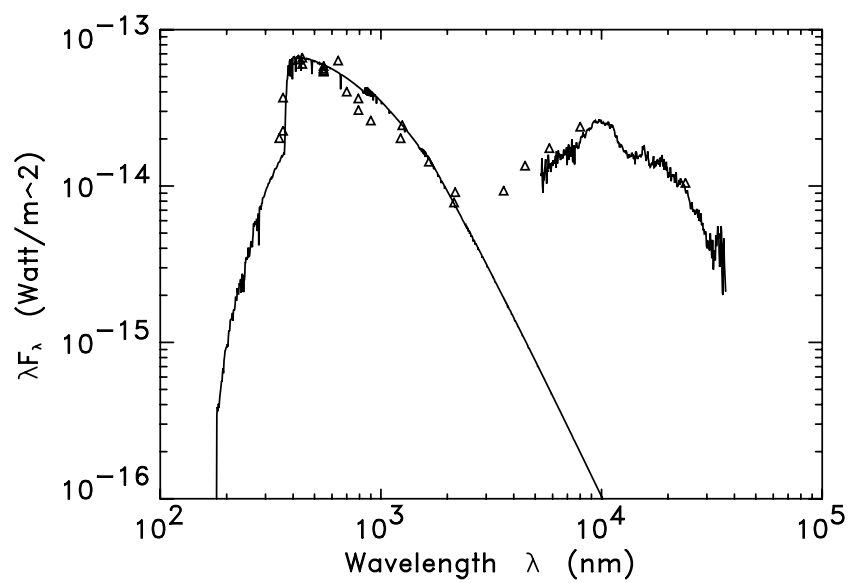

FIG. 8.-Dereddened SED of RV Tauri star MACHO 82.8405.15 (Gielen et al. 2009). Triangles are broadband fluxes. The full line is the associated Kurucz stellar atmosphere model. The SAGE-Spec infrared spectrum is also shown. Thermal emission from amorphous silicates dominates the spectrum but substructure in the $10 \mu \mathrm{m}$ profile as well as the presence of smaller features at longer wavelengths indicate the presence of crystalline silicates (Gielen et al. 2009). This object shows a clear correlation between abundance pattern (Reyniers \& van Winckel 2007) and condensation temperature of species, consistent with reaccretion.

emission longward of $\sim 80 \mu \mathrm{m}$. In order to extract some physical properties of the PAHs and ionic lines in the spectral cube, the spectrum was fitted using an adapted version of PAHFIT (Smith et al. 2007b) that handles both the IRS $(5-40 \mu \mathrm{m})$ and MIPS SED $(70-90 \mu \mathrm{m})$ spectra. To extract abundances of the PAHs and grains populations responsible for the MIR and FIR continuum emission, we used an empirical dust model, DUSTEM (see Bernard et al. 2008, for a description) that is based on the emission model of Désert et al. (1990). We fit the integrated spectrum with the DUSTEM model allowing the radiation field intensity $\left(X_{\mathrm{ISRF}}\right)$, the abundance of the three dust components $\left(Y_{\mathrm{PAH}}\right.$; very small grains, $Y_{\mathrm{VSG}}$; and big grains, $\left.Y_{\mathrm{BG}}\right)$ and the intensity of the NIR continuum to vary simultaneously. The size distribution of the PAHs and BGs remain in the form of a power law as in the solar neighborhood (Désert et al. 1990), but we model the VSGs with a flatter size distribution to account for the flatter shape of the FIR continuum: $n(a) d a \propto a^{\alpha}$ with $\alpha=-1$, where $a$ is the grain radius and $\alpha$ the index of the size distribution of the VSGs. The best fit is shown in Figure 9 along with the SAGE-Spec spectrum normalized to a total dust column density of $10^{20} \mathrm{H} \mathrm{cm}^{-2}$ as derived from the $\mathrm{HI}$ and $\mathrm{CO}$ maps. Figure 9 also shows the photometry points derived from integrating the SAGE-LMC IRAC and MIPS maps over the same region used to extract the spectrum.

The DUSTEM fit results are given in Table 7 and show that the dust abundances derived are somewhat in agreement with the results given in Bernard et al. (2008) for cloud CO-154, indicating that the region selected for spectral mapping is representative of the larger region mapped in the SAGE-LMC 


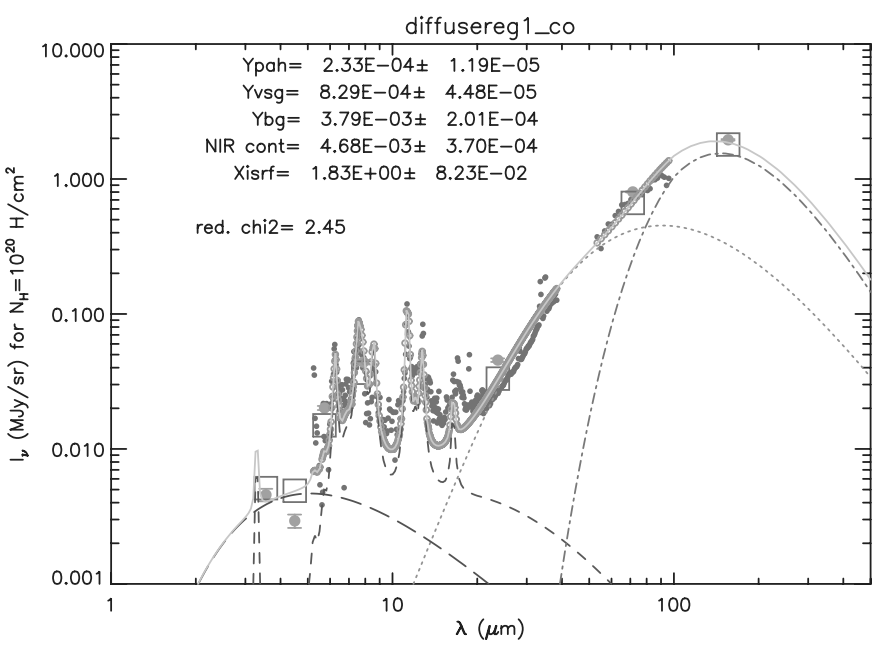

FIG. 9.-Best fit derived using the DUSTEM code for the spectrum toward SSDR 1 (CO-154). The red dots show the IRS and MIPS SED SAGE-Spec spectrum integrated as discussed in the text. The blue dots show the Spitzer photometry integrated in the SAGE data for the same area. All values are normalized to a total column density of $N_{\mathrm{H}}=10^{20} \mathrm{~cm}^{-2}$. The various lines show the contribution of individual dust components: (dot-dash), big grains; (dashed), very small grains; (dashed), PAH; (long dash), NIR continuum. The total model spectrum is shown by the continuous line. The squares show the photometry in the Spitzer bands derived from the model spectrum. The bestfit values for the free parameters shown are those of Table 7. See the electronic edition of the PASP for a color version of this figure.

imaging survey. Note that the large value derived for the NIR continuum probably reflects that the stellar contribution was not removed from the data presented here. This does not affect the fit parameters for the PAH, VSG, and BG component, as the NIR wavelength range is not used to derive the grain properties of those species.

The intensities of the major PAH features derived using PAHFIT are shown in Table 8. Figure 10 shows a comparison between the PAH strength ratios in SSDR 1 with those of other nearby galaxies (Smith et al. 2007b). The ISM for all of these extragalactic spectra contain a range of radiation fields. Although SSDR 1 is toward molecular cloud CO-154, there is clearly ionized gas in the line-of-sight, based on the presence of the [NeIII] 15.5 and [NeII] $12.8 \mu \mathrm{m}$ lines. The ratio of these lines, $[\mathrm{NeIII}] /[\mathrm{NeII}]=0.17$, indicating low-ionization gas, is near the median of the ratios for the extragalactic lines-of-sight studied by Smith et al. (2007b). Nonetheless, we separated the high-ionization lines-of-sight in order to keep the comparison as even as possible. It is evident that the 8.6, 11.3, and $12.6 \mu \mathrm{m}$ features are significantly stronger for SSDR 1 than the average for the sample of nearby galaxies. The relatively bright $11.3 \mu \mathrm{m}$ feature is characteristic of the ISM in the Magellanic Clouds. The features were first detected outside HII regions in the SMC using ISO (Reach et al. 2000) for a region similar in many ways to SSDR 1 considered here; the feature ratios have been interpreted as indicating a different intrinsic set of PAH band strengths in the SMC as compared to those in the Milky Way (Li \& Draine 2002).

\subsubsection{Young Stellar Objects}

Figure 11 shows two examples of embedded YSOs in the SAGE-Spec sample. Both spectra exhibit strong absorption at $15.2 \mu \mathrm{m}$ attributed to the $\mathrm{CO}_{2}$ ice bending mode. These objects also show strong silicate absorption at $10 \mu \mathrm{m}$, as well as emission features due to PAHs, more noticeable at 6,8 , and $11 \mu \mathrm{m}$. The lower spectrum also seems to show an additional broad absorption feature at $6 \mu \mathrm{m}$. This ice band is mainly due to the $\mathrm{O}-\mathrm{H}$ bending mode of water ice, but it is also thought to include contributions of other minor ice species. We have analyzed in detail the $\mathrm{CO}_{2}$ profiles of a sample of massive embedded YSOs identified in the SAGE-Spec survey and from archival data, of which the results are presented in another article (Oliveira et al. 2009), and summarized here. We compute column densities and compare the observed profiles with laboratory profiles available in the literature. The observed profiles show a varied morphology that, when modeled with the help of lab profiles, provides clues on the ice's admixtures and environmental properties, like temperature. We also investigate the properties of the 5-7 $\mu \mathrm{m}$ band. By comparing observed properties in LMC and Galactic sample it is possible to get a handle on metallicity effects on ice chemistry (Oliveira et al. 2009).

TABLE 7

Physical Parameters Derived from the DUSTEM Fit Compared to those Obtained in Bernard et AL. (2008) FOR ClOud CO-154

\begin{tabular}{lccccccc}
\hline \hline & & $Y_{\mathrm{PAH}}\left(10^{-4}\right)$ & $Y_{\mathrm{VSG}}\left(10^{-4}\right)$ & $Y_{\mathrm{BG}}\left(10^{-3}\right)$ & NIR cont $\left(10^{-4} \mathrm{MJY} / \mathrm{sr}\right)$ & $X_{\text {ISRF }}$ & $\chi^{2} / \mathrm{dof}$ \\
\hline SAGE-Spec & $\ldots . .$. & 2.33 & 8.29 & 3.79 & 46.8 & 1.83 & 2.44 \\
CO-154(H I $)$ & $\ldots .$. & 1.78 & 8.25 & 2.85 & 4.99 & 2.59 & 6.61 \\
CO-154(CO) & $\ldots .$. & 0.80 & 2.47 & 0.98 & 15.2 & 3.55 & 7.09 \\
\hline
\end{tabular}

NotE.-The values labeled with "H I" are run for a grain size distribution applicable to the Solar Neighborhood, while the values labeled "CO" are those for case 2 as described by Bernard et al. (2008), in which the same flattened grain size distribution is used for the VSGs as in the present work. 
TABLE 8

Strengths of the Major PAH Features for SSDR 1, Also KnOwn as NANTEN CO CLOUd CO-154

\begin{tabular}{lcc}
\hline \hline Feature Name & Strength $\left(\mathrm{Wm}^{-2} \mathrm{sr}^{-1}\right)$ & Uncertainty $\left(\mathrm{Wm}^{-2} \mathrm{sr}^{-1}\right)$ \\
\hline $6.2 \mu \mathrm{m} \ldots \ldots \ldots \ldots \ldots$ & 1290.8 & 1.2 \\
$7.7 \mu \mathrm{m}$ Complex $\ldots \ldots$. & 3374.8 & 5.2 \\
$8.6 \mu \mathrm{m} \ldots \ldots \ldots \ldots \ldots$. & 1525.4 & 1.4 \\
$11.3 \mu \mathrm{m}$ Complex $\ldots \ldots$ & 2350.9 & 0.7 \\
$12.0 \mu \mathrm{m} \ldots \ldots \ldots \ldots \ldots$ & 674.3 & 0.9 \\
$12.6 \mu \mathrm{m}$ Complex $\ldots \ldots$ & 945.9 & 0.9 \\
$13.6 \mu \mathrm{m} \ldots \ldots \ldots \ldots \ldots$ & 453.6 & 0.8 \\
$14.2 \mu \mathrm{m} \ldots \ldots \ldots \ldots \ldots$ & 49.0 & .8 \\
$16.4 \mu \mathrm{m} \ldots \ldots \ldots \ldots \ldots$ & 171.5 & .4 \\
$17 \mu \mathrm{m}$ Complex $\ldots \ldots \ldots$ & 668.7 & 1.2 \\
$17.4 \mu \mathrm{m} \ldots \ldots \ldots \ldots \ldots$ & 53.1 & 0.4 \\
\hline
\end{tabular}

\subsection{The Nature of Point Sources}

\subsubsection{MIPS SED Point Sources}

Two examples of MIPS SED spectra (van Loon et al. 2010) are shown together with their IRS spectra in Figure 3. These two objects are very different, both in their appearance and in their nature: IRAS $05280-6910$ is an OH/IR star of high luminosity (Wood et al. 1992); it is perhaps the most dust-enshrouded supergiant known to exist in the Magellanic Clouds (van Loon et al. 2005a). We might be dealing with a flattened circumstellar envelope, such as that of WOH G064 (Ohnaka et al. 2008) but in this case viewed edge-on and thus rendering the central star invisible at optical wavelengths. This picture is confirmed by the large optical depth in the silicate features in the IRS range and
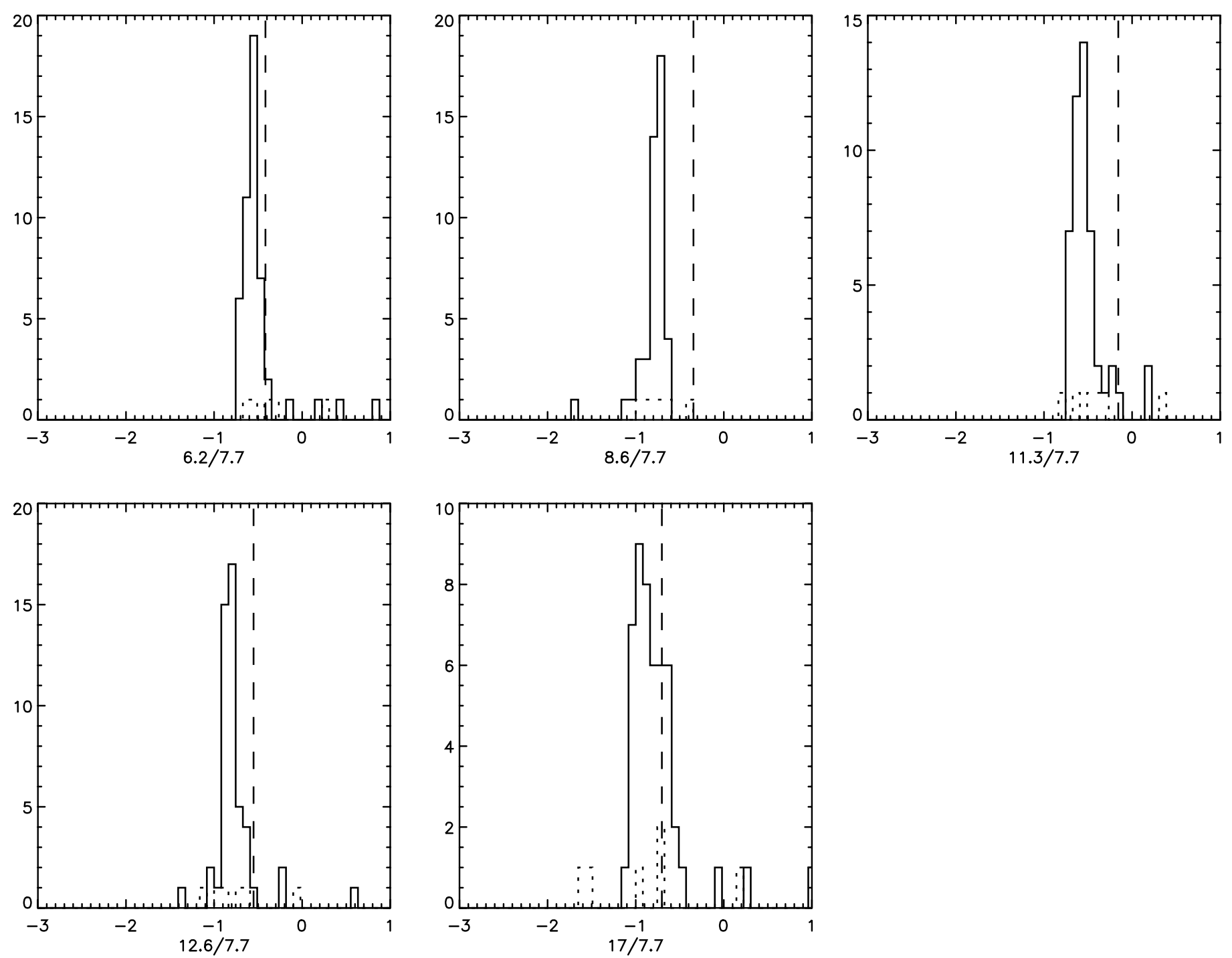

FIG. 10.- - Histograms of the ratios of PAH feature strengths from a sample of nearby galaxies (Smith et al. 2007b). Each ratio is normalized to the $7.7 \mu$ m PAH complex. The solid histograms are for the 50 galaxies in the sample with [Ne III] $<$ [Ne II], as observed for SSDR 1, while the dotted histograms are for the 9 others. The feature ratios for SSDR 1 (CO-154) are indicated with vertical dashed lines. 


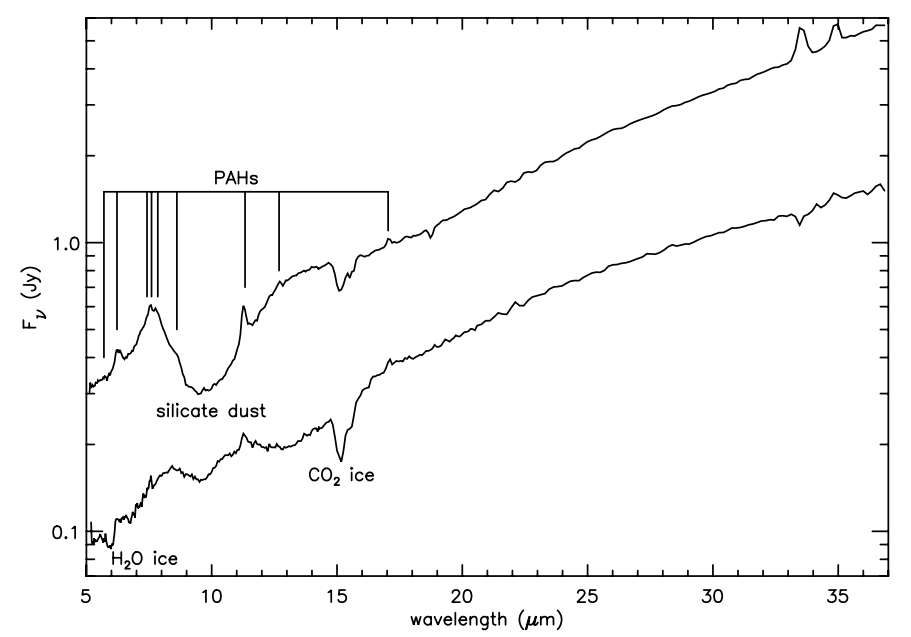

FIG. 11.-Examples of IRS spectra for two embedded YSOs in the LMC (Oliveira et al. 2009). Both objects exhibit a red dust continuum characteristic of the early stages of YSO evolution. Both objects show the broad feature at $10 \mu \mathrm{m}$ associated with silicate dust and PAH emission most noticeably at 6,8 , and $13 \mu \mathrm{m}$. Their spectra also show prominent ice signatures: $\mathrm{CO}_{2}$ ice at $15.2 \mu \mathrm{m}$, and the 5-7 $\mu \mathrm{m}$ ice complex (for the bottom object) that includes among others an important contribution from water ice.

the absence of cold dust in the MIPS SED range. The other object, IRAS 05137-6914 is an ultracompact HII region detected at radio wavelengths (Mathewson et al. 1985; Bojičić et al. 2007). It must harbor a massive young, hot star. The cold dust dominating the cocoon around this young star emits an intense FIR continuum. The excitation and ionization conditions in the gas are traced by the fine-structure line emission of [OI] at $63 \mu \mathrm{m}$ and [OIII] at $88 \mu \mathrm{m}$ in the MIPS SED range; in the IRS range the [SIII] lines at 18 and $33 \mu \mathrm{m}$ are very bright and PAH emission as a consequence of the erosion of small grains is seen. We present a thorough description of the nature of all SAGE-Spec MIPS SED point sources in a related study (van Loon et al. 2010).

\subsubsection{A Background Quasar with Peculiar Properties}

The SAGE-Spec program has also yielded IRS spectra of a number of sources that could not easily be classified based on their broadband colors. Several of these are background sources. One such background source-SAGE 1C J053634.78722658.5-stands out in the IRS spectrum. It has a redshifted spectrum $(z=0.14)$ that exhibits extremely prominent silicate emission features at 10 and $18 \mu \mathrm{m}$. We have analyzed the source and discussed its nature (Hony et al. 2010). We argue that the peculiar IRS spectrum and its corresponding broad wavelength energy distribution are indicative of a quasar. We do not detect any emission from the host galaxy; neither the stellar component in the optical or NIR nor the colder ISM in the FIR (see Fig. 12; Hony et al. 2010), and this may thus be another example of a hostless quasar (Magain et al. 2005).

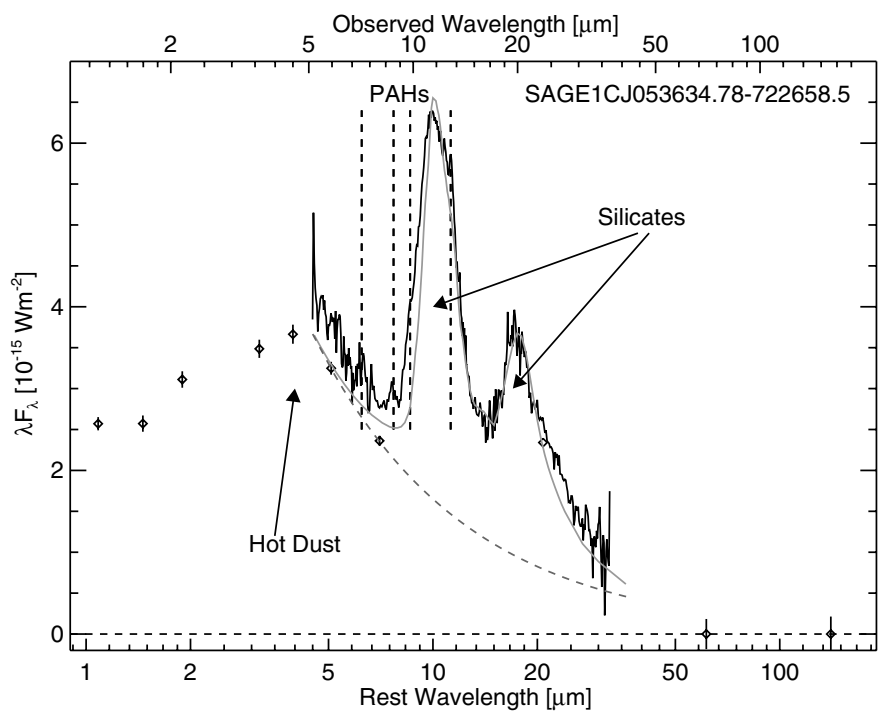

FIG. 12.-Broad wavelength energy distribution of background quasar SAGE 1C J053634.78-722658.5 combined with the remarkable IRS spectrum of the source (Hony et al. 2010). The main contributions that can be identified are the exceptionally strong silicate emission bands at 9.7 and $18 \mu \mathrm{m}$ and the hot continuum peaking near $4 \mu \mathrm{m}$. The weak PAH emission bands have been used to derive the redshift of 0.14 .

\section{OUTLOOK AND CONCLUSIONS}

The SAGE-Spec program provides useful data for understanding the life cycle of gas and dust in galaxies. The extensive data set of IRS and MIPS SED spectroscopy, obtained within the SAGE-Spec program, complemented with archival data, samples the relevant environments and ultimately provides insights on dust mineralogy and gas properties in these environments. Feeding the results back to the original SAGE-LMC data leads to conclusions on stellar populations, allowing us to study the mineralogical dust cycle in the LMC, in combination with the global star formation rate (Whitney et al. 2008; Gruendl \& Chu 2009) and injection rate of stellar mass loss into the ISM (e.g., Matsuura et al. 2009; Srinivasan et al. 2009). An important outcome of the SAGE-Spec program is contributing distinguishing diagnostics to classify sources in the SAGE-LMC point source catalog.

The initial results discussed in the article include the first extragalactic detection of the $21 \mu \mathrm{m}$ feature; the study of crystalline silicates in the disks around RV Tauri stars; the possible detection of a hostless quasar; the analysis of ices toward massive YSOs; and investigations into feature and line ratios in atomic and HII regions to probe physical conditions, such as radiation field and ionization fraction.

True to its legacy status, the SAGE-Spec team has delivered a significant fraction of its reduced data to the scientific community already, with further data deliveries planned in the near future. The unprocessed data have been available to the community in the Spitzer archive from the date of observing. We 
will also deliver enhanced data products, particularly spectral feature maps and source and spectral classifications in those future deliveries.

M. Cohen thanks NASA for supporting his participation in SAGE-Spec through JPL grant 1320707 with UC Berkeley.
B. Sargent, M. Meixner, and B. Shiao were supported for SAGE-Spec through JPL/SSC grant 1310534 with STScI. M. Meixner was additionally supported by NASA NAG512595. R. Szczerba acknowledges support from grant N203 393334 (MNiSW).

\section{REFERENCES}

Alcock, C., et al. 1998, AJ, 115, 1921

Beichman, C. A., Neugebauer, G., Habing, H. J., Clegg, P. E., \& Chester, T. J. 1988, Infrared astronomical satellite (IRAS) catalogs and atlases, Volume 1, Explanatory supplement

Bernard, J.-P., et al. 2008, AJ, 136, 919

Bernard-Salas, J., Peeters, E., Sloan, G. C., Cami, J., Guiles, S., \& Houck, J. R. 2006, ApJ, 652, L29

Bernard-Salas, J., Pottasch, S. R., Gutenkunst, S., Morris, P. W., \& Houck, J. R. 2008, ApJ, 672, 274

Blum, R. D., et al. 2006, AJ, 132, 2034

Bojičić, I. S., Filipović, M. D., Parker, Q. A., Payne, J. L., Jones, P. A., Reid, W., Kawamura, A., \& Fukui, Y. 2007, MNRAS, 378, 1237

Bouchet, P., et al. 2006, ApJ, 650, 212

Buchanan, C. L., Kastner, J. H., Forrest, W. J., Hrivnak, B. J., Sahai, R., Egan, M., Frank, A., \& Barnbaum, C. 2006, AJ, 132, 1890

Buchanan, C. L., Kastner, J. H., Hrivnak, B. J., \& Sahai, R. 2009, AJ, 138, 1597

Cioni, M. R., van der Marel, R. P., Loup, C., \& Habing, H. J. 2000, A\&A, 359, 601

Cohen, M., Wheaton, W. A., \& Megeath, S. T. 2003, AJ, 126, 1090

Dale, D. A., et al. 2007, ApJ, 655, 863

Davies, R. D., Elliott, K. H., \& Meaburn, J. 1976, MmRAS, 81, 89

de Graauw, T., et al. 1996, A\&A, 315, L49

Désert, F.-X., Boulanger, F., \& Puget, J. L. 1990, A\&A, 237, 215

Egan, M. P., Van Dyk, S. D., \& Price, S. D. 2001, AJ, 122, 1844

Fazio, G. G., et al. 2004, ApJS, 154, 10

Feast, M. 1999, PASP, 111, 775

Fouqué, P., et al. 2000, A\&AS, 141, 313

Fukui, Y., et al. 2008, ApJS, 178, 56

Furlan, E., et al. 2006, ApJS, 165, 568

Gaustad, J. E., McCullough, P. R., Rosing, W., \& Van Buren, D. 2001, PASP, 113, 1326

Gielen, C., van Winckel, H., Min, M., Waters, L. B. F. M., \& Lloyd Evans, T. 2008, A\&A, 490, 725

Gielen, C., et al. 2009, A\&A, 508, 1391

Giridhar, S., Lambert, D. L., Reddy, B. E., Gonzalez, G., \& Yong, D. 2005, ApJ, 627, 432

Gordon, K. D., Clayton, G. C., Misselt, K. A., Landolt, A. U., \& Wolff, M. J. 2003, ApJ, 594, 279

Gordon, K. D., Engelbracht, C. W., Rieke, G. H., Misselt, K. A., Smith, J.-D. T., \& Kennicutt, R. C., Jr. 2008, ApJ, 682, 336

Gordon, K. D., et al. 2005, PASP, 117, 503

Green, J. A., et al. 2008, MNRAS, 385, 948

Gruendl, R. A., \& Chu, Y. 2009, ApJS, 184, 172

Gruendl, R. A., Chu, Y.-H., Seale, J. P., Matsuura, M., Speck, A. K., Sloan, G. C., \& Looney, L. W. 2008, ApJ, 688, L9

Higdon, S. J. U., et al. 2004, PASP, 116, 975

Hony, S., et al. 2010, ApJ, submitted

Houck, J. R., et al. 2004, ApJS, 154, 18
Hrivnak, B. J., Volk, K., \& Kwok, S. 2009, ApJ, 694, 1147

Indebetouw, R., et al. 2009, ApJ, 694, 84

Kastner, J. H., Buchanan, C. L., Sargent, B., \& Forrest, W. J. 2006, ApJ, 638, L29

Kastner, J. H., Thorndike, S. L., Romanczyk, P. A., Buchanan, C. L., Hrivnak, B. J., Sahai, R., \& Egan, M. 2008, AJ, 136, 1221

Kennicutt, R. C., Jr., \& Hodge, P. W. 1986, ApJ, 306, 130

Kessler, M. F., et al. 1996, A\&A, 315, L27

Kim, S., Staveley-Smith, L., Dopita, M. A., Sault, R. J., Freeman, K. C., Lee, Y., \& Chu, Y.-H. 2003, ApJS, 148, 473

Lagadec, E., et al. 2007, MNRAS, 376, 1270 2009, MNRAS, 396, 598

Leisenring, J. M., Kemper, F., \& Sloan, G. C. 2008, ApJ, 681, 1557

Leisy, P., Dennefeld, M., Alard, C., \& Guibert, J. 1997, A\&AS, 121,407

Li, A., \& Draine, B. T. 2002, ApJ, 576, 762

Lu, N., et al. 2008, PASP, 120, 328

Maas, T., Van Winckel, H., \& Lloyd Evans, T. 2005, A\&A, 429, 297

Magain, P., Letawe, G., Courbin, F., Jablonka, P., Jahnke, K., Meylan, G., \& Wisotzki, L. 2005, Nature, 437, 381

Massey, P. 2002, ApJS, 141, 81

Mathewson, D. S., Ford, V. L., Tuohy, I. R., Mills, B. Y., Turtle, A. J., \& Helfand, D. J. 1985, ApJS, 58, 197

Matsuura, M., et al. 2006, MNRAS, 371, 415

- 2007, MNRAS, 382, 1889

- 2009, MNRAS, 396, 918

Meixner, M., et al. 2006, AJ, 132, 2268

Misselt, K. A., Clayton, G. C., \& Gordon, K. D. 1999, ApJ, 515, 128

Monet, D. G., et al. 2003, AJ, 125, 984

Ohnaka, K., Driebe, T., Hofmann, K.-H., Weigelt, G., \& Wittkowski, M. 2008, A\&A, 484, 371

Oliveira, J. M., et al. 2009, ApJ, 707, 1269

Pak, S., Jaffe, D. T., van Dishoeck, E. F., Johansson, L. E. B., \& Booth, R. S. 1998, ApJ, 498, 735

Price, S. D., Paxson, C., Engelke, C., \& Murdock, T. L. 2004, AJ, 128,889

Reach, W. T., Boulanger, F., Contursi, A., \& Lequeux, J. 2000, A\&A, 361,895

Reid, W. A., \& Parker, Q. A. 2006, MNRAS, 373, 521

Reyniers, M., \& van Winckel, H. 2007, A\&A, 463, L1

Rieke, G. H., et al. 2004, ApJS, 154, 25

Sandstrom, K. M., Bolatto, A. D., Stanimirović, S., van Loon, J. T., \& Smith, J. D. T. 2009, ApJ, 696, 2138

Seale, J. P., Looney, L. W., Chu, Y.-H., Gruendl, R. A., Brandl, B., Chen, C.-H. R., Brandner, W., \& Blake, G. A. 2009, ApJ, 699, 150

Skrutskie, M. F., et al. 2006, AJ, 131, 1163

Sloan, G. C., Devost, D., Bernard-Salas, J., Wood, P. R., \& Houck, J. R. 2006a, ApJ, 638, 472 
Sloan, G. C., Kraemer, K. E., Matsuura, M., Wood, P. R., Price, S. D., \& Egan, M. P. 2006b, ApJ, 645, 1118

Sloan, G. C., Kraemer, K. E., Wood, P. R., Zijlstra, A. A., BernardSalas, J., Devost, D., \& Houck, J. R. 2008, ApJ, 686, 1056

Sloan, G. C., et al. 2009, Science, 323, 353

Smith, J. D. T., et al. 2007a, PASP, 119, 1133

$$
\text { 2007b, ApJ, 656, } 770
$$

Speck, A. K., Cami, J., Markwick-Kemper, C., Leisenring, J., Szczerba, R., Dijkstra, C., Van Dyk, S., \& Meixner, M. 2006, ApJ, 650, 892

Srinivasan, S., et al. 2009, AJ, 137, 4810

Stanghellini, L., García-Lario, P., García-Hernández, D. A., PereaCalderón, J. V., Davies, J. E., Manchado, A., Villaver, E., \& Shaw, R. A. 2007, ApJ, 671, 1669

Staveley-Smith, L., Kim, S., Calabretta, M. R., Haynes, R. F., \& Kesteven, M. J. 2003, MNRAS, 339, 87

Tappe, A., Rho, J., \& Reach, W. T. 2006, ApJ, 653, 267

van der Marel, R. P., \& Cioni, M. R. L. 2001, AJ, 122, 1807

van Loon, J. T., Marshall, J. R., \& Zijlstra, A. A. 2005a, A\&A, 442, 597

van Loon, J. T., et al. 2005b, MNRAS, 364, L71 . 2010, AJ, 139, 68

van Winckel, H. 2003, ARA\&A, 41, 391
Voors, R. H. M., Waters, L. B. F. M., Morris, P. W., Trams, N. R., de Koter, A., \& Bouwman, J. 1999, A\&A, 341, L67

Waters, L. B. F. M., Trams, N. R., \& Waelkens, C. 1992, A\&A, 262, L37

Werner, M. W., et al. 2004, ApJS, 154, 1

Westerlund, B.E. 1997, The Magellanic Clouds (Cambridge: Cambridge University Press)

Whitney, B. A., et al. 2008, AJ, 136, 18

Williams, R. M., Chu, Y.-H., \& Gruendl, R. 2006, AJ, 132, 1877

Wood, P. R., \& Cohen, M. 2001, in Post-AGB objects as a phase of stellar evolution, eds. Szczerba, R., \& Górny, S. K. (The Netherlands: Kluwer Academic Publishers), 71

Wood, P. R., Whiteoak, J. B., Hughes, S. M. G., Bessell, M. S., Gardner, F. F., \& Hyland, A. R. 1992, ApJ, 397, 552

Woods, P. M., Sloan, G. C., Gordon, K. D., Shiao, B., Kemper, F., \& the SAGE-Spec team 2010, Sage-spectroscopy: The life-cycle of dust and gas in the Large Magellanic Cloud. Data delivery document v2.0, http://data.spitzer.caltech.edu/popular/sage-spec/20100301_ enhanced/docs/SAGESpecDataDelivery2.pdf

Zaritsky, D., Harris, J., \& Thompson, I. 1997, AJ, 114, 1002

Zaritsky, D., Harris, J., Thompson, I. B., \& Grebel, E. K. 2004, AJ, 128,1606

Zijlstra, A. A., et al. 2006, MNRAS, 370, 1961 